\title{
Revisión a algunos geocronómetros radiométricos aplicables al Cuaternario
}

\author{
Juan Pablo Bernal ${ }^{1, *,+}$, Laura E. Beramendi Oroscoํㅜ Karina C. Lugo-Ibarra ${ }^{2}$, Luis Walter \\ Daesslé ${ }^{2}$ \\ ${ }^{1}$ Instituto de Geología, Universidad Nacional Autónoma de México, Ciudad Universitaria, Coyoacán, 04510 México, D.F., México. \\ + Dirección actual: Centro de Geociencias, Universidad Nacional Autónoma de México, Blvd. Juriquilla 3001, 76230 Juriquilla, \\ Querétaro, México. \\ ${ }^{2}$ Instituto de Investigaciones Oceanológicas, Universidad Autónoma de Baja California, Ensenada, Baja California 22830, México. \\ *jpbernal@geociencias.unam.mx
}

\section{Resumen}

El presente artículo describe los fundamentos de los sistemas para dataciones radiométricas de procesos geológicos y ambientales ocurridos en el Cuaternario tardío ( $<500000$ años), específicamente ${ }^{14} \mathrm{C},{ }^{210} \mathrm{~Pb}$ y ${ }^{238} \mathrm{U}_{-}{ }^{234} \mathrm{U}-{ }^{230} \mathrm{Th}$. Se presenta al lector sobre los distintos tipos de especimenes que son susceptibles de ser fechados, así como las distintitas ventajas y limitaciones de cada uno de los sistemas geocronológicos.

Palabras clave: Datación, ${ }^{14} \mathrm{C},{ }^{210} \mathrm{~Pb},{ }^{137} \mathrm{Cs}$, Series de U.

\section{Abstract}

This paper describes the systematics of the main dating systems for geological and environmental processes during the lateQuaternary (<500000 years), specifically ${ }^{14} \mathrm{C},{ }^{210} \mathrm{~Pb}$, and ${ }^{238} \mathrm{U}_{-}^{234} \mathrm{U}^{230} \mathrm{Th}$. A short discussion is presented on the variety of samples amenable to dating, along with the advantages and limitations of the geochronological systems described here.

Keywords: Dating, ${ }^{14} \mathrm{C},{ }^{210} \mathrm{~Pb},{ }^{137} \mathrm{Cs}$, U-Series.

\section{Introducción}

El estudio de procesos climáticos y ambientales ha tomado gran relevancia en los últimos años debido a la serie de implicaciones sociales y económicas que representan la contaminación ambiental y el cambio climático. De esta manera, el estudio de los distintos registros ambientales (por ejemplo núcleos de sedimento, perfiles edáficos, anillos de árbol, etc.,) ha permitido establecer las condiciones y variabilidad naturales de diversos indicadores ambientales. Asimismo, han permitido identificar los principales efectos forzadores de la variablidad natural y, por lo tanto, discernir entre efectos de origen natural y antropogénico. Lo anterior no sería posible sin una metodología que no sólo permita establecer una cronología precisa de los procesos involucrados para saber cuando sucedieron dichos eventos, sino también comparar y/o complementar con registros contemporáneos y, por lo tanto, llegar a establecer el alcance regional de los procesos bajo estudio.

El establecimiento de una cronología de eventos para un registro (paleo)ambiental se puede llevar a cabo de distintas maneras. Por ejemplo, debido a su naturaleza, el 
estudio de esqueletos de corales y anillos de árbol permite establecer la cronología a partir del conteo de bandas anuales (p.e., Baker et al., 1993; McCulloch et al., 2003). Este procedimiento puede ser también aplicado al estudio de algunas estalagmitas, sedimentos laminados, núcleos de hielo, esqueletos de corales e, incluso, algunos fósiles. Lo anterior requiere de manera irrestricta de, al menos, una fecha conocida de manera independiente a partir de la cual contar los correspondientes estratos, así como un conocimiento detallado del proceso de bandeamiento.

La necesidad de contar con series de tiempo con estricto control cronológico independiente ha llevado al desarrollo de distintos métodos de datación basados, principalmente, en el decaimiento radioactivo de isótopos de origen natural. De éstos, posiblemente el más comúnmente referido es el ${ }^{14} \mathrm{C}$. Sin embargo, el reciente desarrollo de instrumental de alta sensibilidad ha permitido medir con alta precisión la abundancia de distintos isótopos cuyas vidas medias cortas y su baja abundancia dificultó en el pasado la cuantificación confiable y, por lo tanto, su uso como geocronómetros.

El presente documento tiene como objetivo presentar al lector los fundamentos de algunos de los métodos de datación utilizados con mayor frecuencia en estudios paleoambientales, de manera que se provea de una base para la comprensión de documentos técnicos más avanzados, así como para comprender los criterios de selección y recolección de muestras.

\section{Datación por Radiocarbono}

La datación por Radiocarbono $\left({ }^{14} \mathrm{C}\right)$ es uno de los métodos más utilizados para generar cronologías del Cuaternario tardío (50000 años) en ámbitos de varias disciplinas como la arqueología, la geología, la paleontología, la paleoclimatología y la vulcanología, entre otras. Si bien el uso del ${ }^{14} \mathrm{C}$ como herramienta para fechar muestras de interés arqueológico fue el motor inicial para el desarrollo de la técnica, su aplicación en Ciencias de la Tierra es muy amplia. Específicamente, en estudios paleoambientales es crucial poder referir a la escala temporal cambios ambientales estudiados en diferentes registros y distribuidos en diferentes latitudes, y es precisamente de radiocarbono la cronología con la que se han producido la mayoría de las reconstrucciones climáticas para el Holoceno y el Pleistoceno tardío (Bard, 1998; Lowe y Walker, 2000; Hajdas, 2009).

\subsection{Formación y decaimiento $\operatorname{del}{ }^{14} \mathrm{C}$}

$\mathrm{El}{ }^{14} \mathrm{C}$ se forma principalmente por la reacción entre los neutrones resultantes de la radiación cósmica y los átomos de nitrógeno del aire que se lleva a cabo en la atmósfera superior, de acuerdo a

$$
\mathrm{n}+{ }_{7}^{14} \mathrm{~N} \rightarrow{ }_{6}^{14} \mathrm{C}+\mathrm{p}
$$

donde $\mathrm{n}$ y $\mathrm{p}$ representan neutrones y protones, respectivamente. La tasa global de formación de radiocarbono no se conoce con exactitud, sin embargo se sabe que varía como consecuencia tanto de cambios en la incidencia de los rayos cósmicos como en el campo magnético terrestre y la actividad solar (Bronk Ramsey, 2008). El ${ }^{14} \mathrm{C}$ reacciona con el oxígeno para formar ${ }^{14} \mathrm{CO}_{2}$, que baja a la tropósfera y se mezcla con el ${ }^{12} \mathrm{CO}_{2}$ y el ${ }^{13} \mathrm{CO}_{2}$ entrando en el ciclo del carbono (Figura 1).

Al ser un isótopo radiactivo, el radiocarbono decae de manera espontánea emitiendo una partícula beta negativa y transformándose en un átomo de ${ }^{14} \mathrm{~N}$, de acuerdo a la siguiente reacción:

$$
{ }^{14} C \rightarrow{ }^{14} N+\beta^{-}
$$

este proceso ocurre con una vida media de $5730 \pm 40$ años (Godwin, 1962).
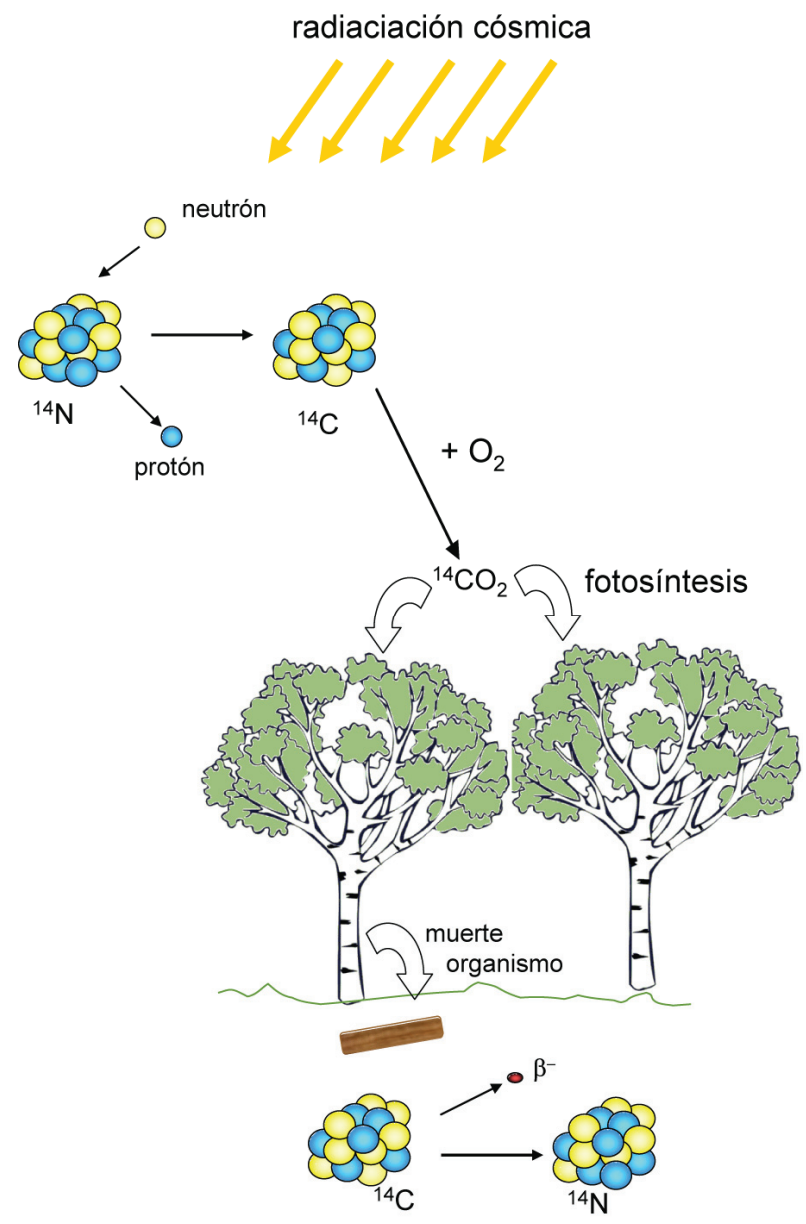

Figura 1. El ${ }^{14} \mathrm{C}$ en la naturaleza. Su formación en las altas capas de la atmósfera, oxidación para formar $\mathrm{CO}_{2}$ e incorporación a la biosfera. Finalmente, su decaimiento por emisión de partículas $\beta$-. 


\section{$2.2 \mathrm{El}^{14} \mathrm{C}$ en los organismos}

La biósfera asimila el ${ }^{14} \mathrm{C}$ atmosférico a través de la fotosíntesis de las plantas terrestres y del fitoplancton, fijando el $\mathrm{CO}_{2}$ con la proporción atmosférica, o acuática para el caso de las algas, de ${ }^{14} \mathrm{C}$; sin embargo, durante la fotosíntesis se da un fraccionamiento isotópico y en consecuencia la concentración de ${ }^{14} \mathrm{C}$ de las plantas difiere de la concentración atmosférica, haciendo necesaria una corrección (ver sección de Edades calibradas). Por otra parte, los animales asimilan ${ }^{14} \mathrm{C}$ a través de la cadena trófica.

Los procesos metabólicos de los seres vivos mantienen la cantidad de ${ }^{14} \mathrm{C}$ prácticamente constante ya que el número de átomos de ${ }^{14} \mathrm{C}$ que pierden los tejidos vivos por decaimiento radiactivo es continuamente reemplazado por medio de la fotosíntesis, en el caso de plantas, y por la alimentación (ingesta de plantas o tejido animal) en el caso de animales. Cuando los procesos metabólicos cesan con la muerte del organismo, la cantidad de ${ }^{14} \mathrm{C}$ empieza a disminuir exponencialmente por el decaimiento radiactivo.

2.3 El modelo de datación y las edades convencionales de ${ }^{14} \mathrm{C}$

Midiendo la concentración de ${ }^{14} \mathrm{C}$ que contiene una muestra se puede entonces calcular el tiempo que ha pasado desde que el organismo dejó de asimilar carbono (muerte del organismo) a través de la siguiente ecuación

$$
\mathrm{t}=-8033 \ln \left(\mathrm{A}_{\mathrm{m}} / \mathrm{A}_{0}\right)
$$

Ecuación 1

donde $t$ es la edad de la muestra en años, $A_{m}$ es la concentración (o actividad) de ${ }^{14} \mathrm{C}$ de la muestra de interés, $\mathrm{A}_{0}$ la Actividad inicial (al momento de la muerte y en equilibrio con la atmósfera) que, por convención, equivale a la concentración de ${ }^{14} \mathrm{C}$ atmosférico de 1950, y 8033 es el inverso de la constante de decaimiento $(\ln 2 / \mathrm{T}$, donde $\mathrm{T}$ = 5568 años, tiempo de vida media).

Este modelo considera, por un lado, que la concentración atmosférica de ${ }^{14} \mathrm{C}$ ha sido constante a lo largo de la escala de tiempo del radiocarbono e igual a la de $1950 \mathrm{y}$, por el otro lado, utiliza la primera determinación de vida media del ${ }^{14} \mathrm{C}$ equivalente a 5568 años (Libby, 1955). Además, considera que el ${ }^{14} \mathrm{C}$ se distribuye de manera homogénea en todos los reservorios de carbono y que no se tienen procesos de fraccionamiento isotópico. Lo anterior hace que las edades calculadas con este modelo se desvíen de la edad verdadera de la muestra, por lo que se deben corregir o calibrar.

Para corregir el error introducido por el fraccionamiento isotópico que pudo haber ocurrido desde el proceso de formación de la muestra hasta el proceso de preparación para el análisis, es necesario determinar la relación ${ }^{13} \mathrm{C} /{ }^{12} \mathrm{C}$ de la muestra, expresada en $\delta^{13} \mathrm{C}$ relativa al VPDB (Vienna
Pee-Dee Belemnite), y se estima que el fraccionamiento del ${ }^{14} \mathrm{C}$ es del doble que para el ${ }^{13} \mathrm{C}$ (Craig, 1954).

Debido a que cuando se determinó con mayor precisión una vida media del ${ }^{14} \mathrm{C}$ de 5730 años ya se habían reportado miles de edades de ${ }^{14} \mathrm{C}$, se decidió por convención seguir utilizando la vida media calculada por Libby (1955), con la finalidad de mantener la congruencia con las edades calculadas previamente (Stuiver y Polach, 1977). De esta manera, las edades calculadas a partir de la Ecuación 1 y corregidas por fraccionamiento isotópico se conocen como edades convencionales y se expresan en años antes del presente (a.P. o BP, por sus siglas en inglés), siendo el presente el año 1950.

\subsection{Edades calibradas}

Debido a las fluctuaciones en la concentración atmosférica de ${ }^{14} \mathrm{C}$ y a que la vida media reconocida como más confiable es ligeramente mayor, una edad convencional no corresponde a la edad verdadera de la muestra y, en consecuencia, es necesario realizar lo que se conoce como calibración. Para calibrar una edad convencional es necesario conocer los niveles atmosféricos de ${ }^{14} \mathrm{C}$ a lo largo de la escala temporal del radiocarbono, lo cual se ha logrado gracias al análisis de anillos de crecimiento de árbol, corales y sedimentos varvados de origen marino que han sido fechados con métodos alternativos al radiocarbono, como la dendrocronología en el caso de los anillos de árbol y la datación por U/Th en el caso de los corales. Esto ha permitido construir curvas de calibración que muestran la relación entre la edad de ${ }^{14} \mathrm{C}$ y la edad absoluta a través del patrón de variación de los niveles de ${ }^{14} \mathrm{C}$ atmosférico (Reimer et al., 2004). El resultado de la calibración corresponde a uno o más intervalos del calendario con probabilidad de que la muestra haya dejado de fijar carbono. De esta manera, una edad calibrada puede corresponder a uno o más intervalos, que pueden abarcar incluso varias décadas, debido a la manera en que ha variado la concentración atmosférica del ${ }^{14} \mathrm{C}$ a lo largo del tiempo (Figura 2).

\subsection{Utilizando la datación por ${ }^{14} \mathrm{C}$}

El primer paso para poder construir cronologías de ${ }^{14} \mathrm{C}$ es la correcta selección de muestras. La datación por radiocarbono se puede utilizar para fechar muestras de origen orgánico. Entre los ejemplos más comunes están restos vegetales, carbón, huesos, materia orgánica en suelos y sedimentos, conchas y corales. En algunos casos también es posible datar muestras inorgánicas, como los carbonatos, pero siempre teniendo en cuenta el posible origen del carbono contenido en la muestra, ya que la presencia de carbono de origen geológico puede hacer que la edad aparente de la muestra sea considerablemente mayor que la edad real (momento en que se precipitó el carbonato). A esta diferencia se le conoce como edad del reservorio y es posible determinarla con ayuda de la datación por U/Th 

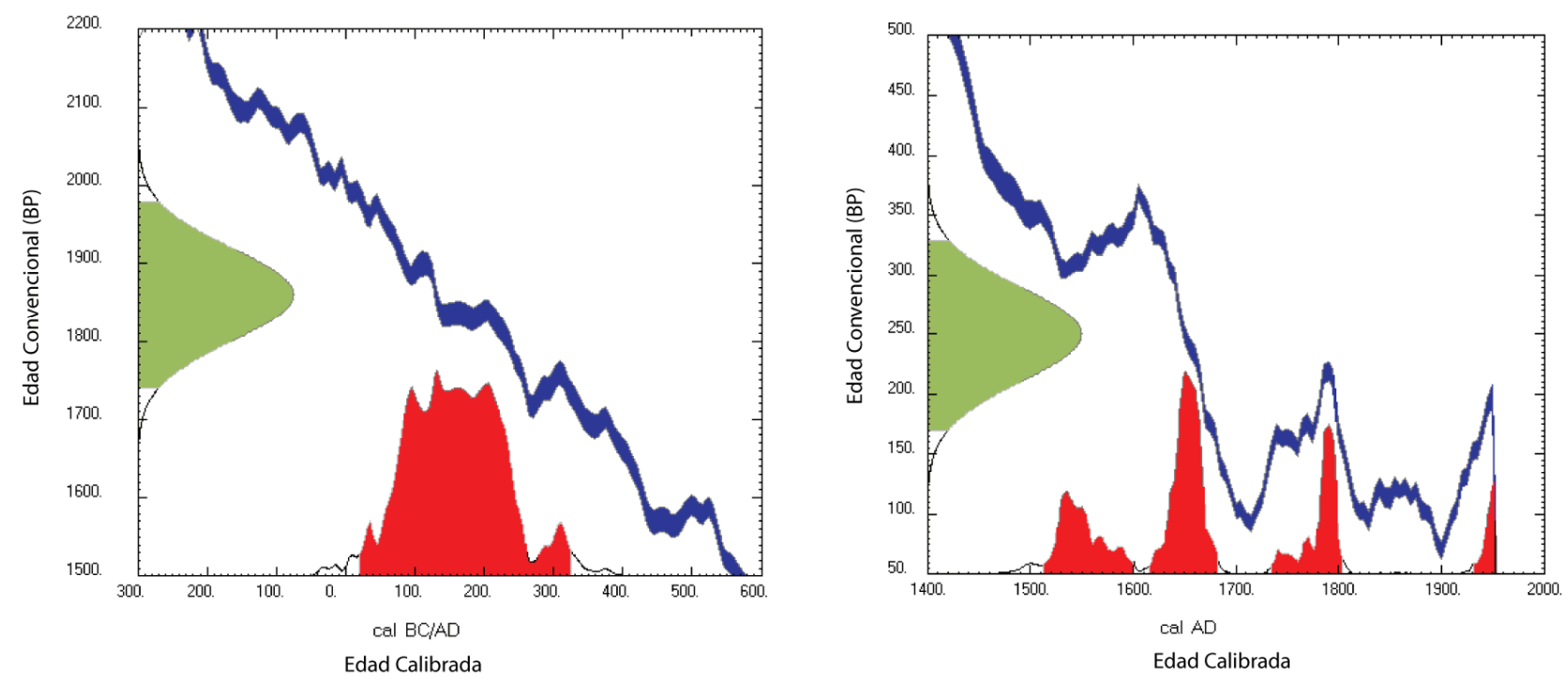

Figura 2. Calibración de edades convencionales de ${ }^{14} \mathrm{C}$. En el eje vertical se muestra la edad convencional, representada como una distribución normal, y en el eje horizontal la edad calibrada, que puede corresponder a uno (izquierda) o más intervalos (derecha).

(Srdoc et al., 1994; Horvatincic et al., 2000).

Es fundamental tener clara la relación que existe entre la muestra y el evento que se desea datar. La fecha calculada por radiocarbono es indicativa del momento en el que la muestra dejó de intercambiar carbono con la atmósfera. Generalmente se seleccionan muestras que provienen de un solo organismo y así es posible determinar el momento en que dicho organismo dejó de fijar carbono sin la incertidumbre de mezclar carbono que haya sido aislado de la atmósfera en momentos diferentes. En algunos casos, sin embargo, es necesario mezclar a una comunidad de organismos para contar con la cantidad necesaria de material para el análisis, asumiendo que todos dejaron de fijar carbono al mismo tiempo. Tal es el caso para diatomeas, foraminíferas y corales.

Es importante evitar y eliminar cualquier tipo de contaminación, tanto la proveniente del ambiente en el que haya estado depositada la muestra, como la introducida al momento de recolección de la muestra. Cualquier contaminación que contenga carbono puede alterar la fecha de radiocarbono, dando resultados significativamente más antiguos o más jóvenes dependiendo del tipo y cantidad de contaminación (Tabla 1).

Un alto porcentaje de las dataciones de radiocarbono se realizan en madera y carbón de madera, pues estos materiales son considerados óptimos por su alto contenido de carbono y sus propiedades químicas, que permiten aplicar procedimientos de pre-tratamiento agresivos para poder eliminar cualquier tipo de contaminación, sin el riesgo de perder una cantidad importante de muestra (Taylor, 1987; Hedges, 1992). Sin embargo, es importante tener en cuenta que no siempre la madera será contemporánea con el evento que se pretende fechar, pues el carbono de la muestra pudo
Tabla 1. Efecto de contaminación con carbono de diferentes edades sobre la edad de ${ }^{14} \mathrm{C}$ de una muestra (adaptado de Bronk Ramsey, 2008).

\begin{tabular}{cccccc}
\hline Tipo de & \multicolumn{5}{c}{ Edad de ${ }^{14} \mathrm{C}$ de la muestra (años) } \\
\cline { 2 - 6 } contaminación & 0 & 5000 & 10000 & 20000 & 40000 \\
\hline $\begin{array}{c}1 \% \text { de C } \\
\text { moderno } \\
1 \% \text { de C }\end{array}$ & 0 & -70 & -200 & -840 & -7200 \\
$\begin{array}{c}\text { geológico } \\
\text { \% de C 1000 } \\
\text { años más } \\
\text { joven }\end{array}$ & 80 & 80 & 80 & 80 & 80 \\
\hline
\end{tabular}

n. a. $=$ no aplica.

haber sido aislado de la atmósfera mucho tiempo antes de que sucediera el evento de interés. Al error que esto introduce se le conoce como "edad inherente" que es la suma del error que introduce la "edad de crecimiento" al datar anillos internos de árboles muy longevos y la "edad de almacenamiento" que está relacionada con el tiempo que pudo haber transcurrido entre que el árbol murió y el evento a fechar (McFadgen et al., 1994). Por lo anterior se recomienda que en caso de muestrear restos vegetales, tanto carbonizados como no carbonizados, se seleccionen de ser posible especies o elementos de vida corta (ramas, troncos pequeños, hojas) o en caso de encontrar troncos grandes muestrear únicamente los anillos externos, que tendrán una edad más cercana al momento de la muerte del árbol.

Algunos procesos que pueden incorporar ${ }^{14} \mathrm{C}$ "moderno" incluyen el crecimiento de hongos y/o bacterias en muestras húmedas. Lo anterior puede generar que la edad de radiocarbono resulte significativamente más joven que la edad real de la muestra. Para evitar la incorporación 
de carbono "moderno", es ampliamente recomendable almacenar las muestras a datar en un amiente frío y seco, que evite el crecimiento de comunidades de algas y bacterias.

\subsection{Metodologías analíticas}

Existen tres técnicas de análisis para la determinación de ${ }^{14} \mathrm{C}$ : el Conteo Proporcional de Gases, la Espectrometría de Centelleo Líquido y la Espectrometría de Aceleración de Masas. Las dos primeras se denominan técnicas radiométricas porque determinan el decaimiento radioactivo del ${ }^{14} \mathrm{C}$ de la muestra relativo a la cantidad de carbono total, mientras que la tercera cuenta directamente el número relativo de átomos de ${ }^{14} \mathrm{C}$ en la muestra utilizando un acelerador de partículas. Las dos técnicas que más se utilizan en la actualidad son la Espectrometría de Centelleo Líquido (LSC, por sus siglas en inglés) y la Espectrometría de Aceleración de Masas (AMS, por sus siglas en inglés). No se van a revisar los fundamentos de ambos métodos en este trabajo, sin embargo es importante mencionar las ventajas y desventajas de cada uno para que el lector pueda decidir qué método le es más conveniente según el tipo, cantidad de muestra y precisión necesarias.

La primera diferencia entre ambos métodos es el tamaño de la muestra. Para LSC se requieren muestras relativamente grandes (que contengan mínimo $1 \mathrm{~g}$ de carbono después de la limpieza), mientras que para AMS es posible obtener un resultado confiable con solamente $50 \mu \mathrm{g}$ para muestras modernas y $1000 \mu \mathrm{g}$ para muestras $>10000$ años, por lo que el segundo método abre la posibilidad de datar una gran diversidad de materiales como polen, diatomeas y fitolitos. Sin embargo, manejar muestras tan pequeñas tiene el inconveniente de que son más susceptibles de contaminarse tanto en el muestreo como en el laboratorio y el efecto de dicha contaminación puede ser significativo. Además, tener una muestra tan pequeña también puede resultar en que ésta no sea realmente representativa del evento que se desea datar.

La segunda diferencia entre LSC y AMS radica en el tiempo de análisis. Debido a que el decaimiento radiactivo es un proceso espontáneo, en LSC es necesario analizar la muestra por largos periodos de tiempo, típicamente 2500 minutos y hasta 10000 minutos para muestras $>30000$ años, para obtener un resultado estadísticamente confiable y una precisión aceptable; mientras que en AMS se requiere de un tiempo de análisis corto, típicamente 5 minutos y máximo 20 para muestras $>25000$ años (Hogg et al., 2006). Otra diferencia entre ambos métodos es la estabilidad del análisis, que se ve reflejada en la precisión. La tecnología AMS lleva pocas décadas de desarrollo y los instrumentos son aún complejos, presentan poca estabilidad y por consecuencia una muestra no puede ser medida por largos periodos limitando así la precisión (Bronk Ramsey, 2008). Por el otro lado, los equipos de LSC son muy estables, permitiendo analizar las muestras por largos periodos de tiempo de tal manera que si se realiza un conteo extendido
(5000 y 10000 minutos), y se tiene cantidad suficiente de muestra, se pueden llegar a tener precisiones de $\pm 0.32 \%$ ( \pm 16 años) para muestras $<5000$ años (Takahashi et al., 2009).

\subsection{Interpretación de la Edad de ${ }^{14} \mathrm{C}$}

Una vez que se tiene el resultado de la relación ${ }^{14} \mathrm{C} /{ }^{12} \mathrm{C}$ de la muestra, corregida por fraccionamiento isotópico, es necesario compararlo con la relación ${ }^{14} \mathrm{C} /{ }^{12} \mathrm{C}$ atmosférica de 1950, de manera que, utilizando la Ecuación 1, se pueda estimar la edad convencional de la muestra. El siguiente paso es realizar la calibración que, como se detalló anteriormente, consiste en comparar la edad convencional de la muestra con la curva de calibración construida con muestras de edad conocida. Es hasta que se realiza la calibración que se puede evaluar la edad de la muestra en términos absolutos, a pesar de que el proceso de calibración puede arrojar resultados con muy baja precisión calendárica, independientemente de la precisión con que se haya determinado la relación ${ }^{14} \mathrm{C}$ $/{ }^{12} \mathrm{C}$, debido a la variabilidad de la concentración del ${ }^{14} \mathrm{C}$ a lo largo del tiempo.

Finalmente, para poder interpretar la edad calibrada es necesario tener clara la relación entre la muestra y el evento de interés, pues la edad calibrada nos va a dar el momento en que se fijó el carbono presente en la muestra, por lo que hay que entender bien cómo se relaciona el organismo del que proviene la muestra con el contexto geológico o arqueológico que se está estudiando.

\section{Datación por desequilibrio en la cadena de decaimiento de ${ }^{238} \mathbf{U}$}

El estudio de los distintos isótopos que componen la cadena de decaimiento del ${ }^{238} \mathrm{U}$ data desde los primeros estudios sobre radioactividad a finales del siglo XIX, encaminados hacia la caracterización de los distintitos tipos de radiación emitidas por los concentrados de pechblenda. Sin embargo, a pesar de que fueron los primeros isótopos en ser identificados y caracterizados, su aplicación al campo de la ciencias de la Tierra se vio limitado debido a la dificultad analítica para cuantificarlos con precisión, característica intrínseca de su baja abundancia y corta vida media. Durante gran parte del siglo XX, la única manera de cuantificar estos isótopos fue utilizando espectroscopia $\alpha$ de los isótopos, o $\gamma$ de los isótopos hijos. Sin embargo, debido a la baja actividad de los isótopos más abundantes, y a la poca abundancia de los isótopos más activos, era necesario utilizar grandes cantidades de muestra y tiempos de análisis largos, incluso semanas, por muestra, por lo que su cuantificación era larga, tediosa, y durante mucho tiempo limitada al análisis de muestras grandes.

El surgimiento de la espectrometría de masas durante el primer tercio del siglo XX, permitió el análisis de algunos de estos isótopos hasta mediados de los 80 's, cuando los isótopos más abundantes de la cadena de decaimiento 
$\left({ }^{238} \mathrm{U}-{ }^{234} \mathrm{U}\right.$ y $\left.{ }^{230} \mathrm{Th}\right)$ pudieron ser cuantificados de manera robusta por espectrometría de masas con ionización térmica (Edwards et al., 1987). Por otra parte, el desarrollo de detectores de centelleo de bajo fondo ha permitido cuantificar con mayor precisión los isótopos menos abundantes pero de mayor actividad en muestras cada vez más reducidas.

3.1 Decaimiento radioactivo en serie y sus expresiones matemáticas

En un sistema donde un isótopo A que decae de manera radioactiva para formar $\mathrm{B}$, el cual a su vez decae para formar C, es decir:

$$
\mathrm{A} \stackrel{\lambda_{\mathrm{A}}}{\longrightarrow} \mathrm{B} \stackrel{\lambda_{\mathrm{B}}}{\longrightarrow} \mathrm{C} \stackrel{\lambda_{\mathrm{C}}}{\longrightarrow} \ldots
$$

la tasa de decaimiento de A está dado por:

$$
-\frac{\mathrm{dN}_{\mathrm{A}}}{\mathrm{dt}}=\lambda_{\mathrm{A}} \mathrm{N}_{\mathrm{A}}
$$

Ecuación 2

donde $\lambda_{\mathrm{A}}$ es la constante de decaimiento de $\mathrm{A} \mathrm{y} \mathrm{N}_{\mathrm{A}}$ el número de átomos de $\mathrm{A}$ al tiempo t. La resolución de la Ecuación 2 como $\mathrm{N}_{\mathrm{A}}=\mathrm{f}(\mathrm{t}) \mathrm{da}$ :

$$
\mathrm{N}_{\mathrm{A}}=\mathrm{N}_{\mathrm{A}}^{0} \mathrm{e}^{-\lambda_{\mathrm{A}} \mathrm{t}}
$$

Ecuación 3

Donde $\mathrm{N}^{0}$ corresponde a la cantidad inicial de A. La tasa de decaimiento de B, en cambio, está dada por la tasa a la que es formado por A, así como por su propia tasa de decaimiento:

$$
\frac{\mathrm{dN}_{\mathrm{B}}}{\mathrm{dT}}=\lambda_{\mathrm{A}} \mathrm{N}_{\mathrm{A}}-\lambda_{\mathrm{B}} \mathrm{N}_{\mathrm{B}}
$$

Ecuación 4

Sustituyendo la Ecuación 3 en la Ecuación 4 y rearreglando:

$$
\frac{\mathrm{dN}_{\mathrm{B}}}{\mathrm{dT}}+\lambda_{\mathrm{B}} \mathrm{N}_{\mathrm{B}}-\lambda_{\mathrm{A}} \mathrm{N}_{\mathrm{A}}^{0} \mathrm{e}^{-\lambda_{\mathrm{A}} \mathrm{t}}=0
$$

Ecuación 5

La cual es una ecuación diferencial lineal de primer grado, cuya solución es:

$$
\mathrm{N}_{\mathrm{B}}=\frac{\lambda_{\mathrm{A}}}{\lambda_{\mathrm{B}}-\lambda_{\mathrm{A}}} \mathrm{N}_{\mathrm{A}}^{0}\left(\mathrm{e}^{-\lambda_{\mathrm{A}} \mathrm{t}}-\mathrm{e}^{-\lambda_{\mathrm{B}} \mathrm{t}}\right)+\mathrm{N}_{\mathrm{B}}^{0} \mathrm{e}^{-\lambda_{\mathrm{B}} \mathrm{t}}
$$

Ecuación 6
El primer término de la ecuación describe el decaimiento de A para formar B, mientras que el segundo término describe el decaimiento radioactivo de B. Idealmente, el segundo término de la Ecuación 6 es 0 o muy cercano a 0 . Para el caso de la serie de decaimiento del U, sin embargo, es necesario considerar que el isótopo $\mathrm{C}$, no corresponde a un isótopo estable, sino que también es radioactivo. De esta manera $\mathrm{N}_{\mathrm{C}}$ se describe de la siguiente manera:

$$
\begin{gathered}
\mathrm{N}_{\mathrm{C}}=\lambda_{\mathrm{A}} \lambda_{\mathrm{B}} \mathrm{N}_{\mathrm{A}}^{0}\left(\frac{\mathrm{e}^{-\lambda_{\mathrm{A}} \mathrm{t}}}{\left(\lambda_{\mathrm{B}}-\lambda_{\mathrm{A}}\right)\left(\lambda_{\mathrm{C}}-\lambda_{\mathrm{A}}\right)}\right)+\lambda_{\mathrm{A}} \lambda_{\mathrm{B}} \mathrm{N}_{\mathrm{A}}^{0}\left(\frac{\mathrm{e}^{-\lambda \mathrm{Bt}}}{\left(\lambda_{\mathrm{A}}-\lambda_{\mathrm{B}}\right)\left(\lambda_{\mathrm{C}}-\lambda_{\mathrm{B}}\right)}\right)+\ldots \\
\ldots+\lambda_{\mathrm{A}} \lambda_{\mathrm{B}} \mathrm{N}_{\mathrm{A}}^{0}\left(\frac{\mathrm{e}^{-\lambda_{\mathrm{C}} \mathrm{t}}}{\left(\lambda_{\mathrm{A}}-\lambda_{\mathrm{C}}\right)\left(\lambda_{\mathrm{B}}-\lambda_{\mathrm{C}}\right)}\right)
\end{gathered}
$$

Ecuación 7

La resolución detallada de las ecuaciones de decaimiento es descrita y generalizada en Bourdon et al. (2003).

La figura 3 muestra la variación de las abundancias de A, $\mathrm{B}$ y $\mathrm{C}$ de acuerdo a las Ecuaciones 3, 6 y 7 para el caso en el que los tres isótopos tienen vidas medias cortas y similares.

Una característica de los sistemas basados en el decaimiento de varios isótopos es que, dado el tiempo suficiente, la tasa de formación de los isótopos es igual a la tasa de decaimiento, por lo que no se aprecia un cambio considerable en la abundancia absoluta y relativa de los isótopos. Esto se ejemplifica en las Figuras 3B y $3 \mathrm{C}$, en donde se muestra la cantidad de isótopos de ${ }^{234} \mathrm{U}$ y ${ }^{230} \mathrm{Th}$ en función del tiempo, los cuales se forman a partir del decaimiento de ${ }^{238} \mathrm{U}$. Se puede demostrar que:

$$
\begin{aligned}
& \frac{\mathrm{N}_{\mathrm{B}}}{\mathrm{N}_{\mathrm{A}}} \rightarrow \frac{\lambda_{\mathrm{A}}}{\lambda_{\mathrm{B}}} \text { si } \mathrm{t} \rightarrow \infty \\
& \frac{\mathrm{N} \text {, por lo tanto }}{\mathrm{N}_{\mathrm{B}} \times \lambda_{\mathrm{B}}} \rightarrow 1 \text { si } \mathrm{t} \rightarrow \infty
\end{aligned}
$$

Ecuación 8

Donde $\mathrm{N} \times \lambda$ se denomina actividad del isótopo correspondiente. Lo anterior implica que, transcurrido el tiempo suficiente, la abundancia relativa de los isótopos no cambiará, como se ilustra en la Figura 3C, donde es posible ver que transcurridos $\sim 10^{6}$ y $\sim 450000$ años, la relaciones $\mathrm{R} 1\left({ }^{234} \mathrm{U} /{ }^{238} \mathrm{U}\right)$ y $\mathrm{R} 2\left({ }^{230} \mathrm{Th} /{ }^{238} \mathrm{U}\right)$, respectivamente, se mantienen constantes e iguales a la relación de constantes de decaimiento (Ecuación 8). Cuando se alcanza el estado en el que las abundancias relativas de los isótopos no cambian, se dice que el sistema ha alcanzado "equilibrio secular". En términos prácticos, un sistema se encuentra en equilibrio secular cuando la diferencia entre la relación isotópica $\mathrm{N}_{\mathrm{B}}$ / $\mathrm{N}_{\mathrm{A}}$ y la composición isotópica esperada para el equilibrio secular $\left(\lambda_{A} / \lambda_{B}\right)$ es menor a la incertidumbre de la medición, lo cual ocurre típicamente después de haber transcurrido unas 7 a 10 vidas medias del isótopo más inestable (Dickin, 1995). De esta manera, las dataciones utilizando estos 

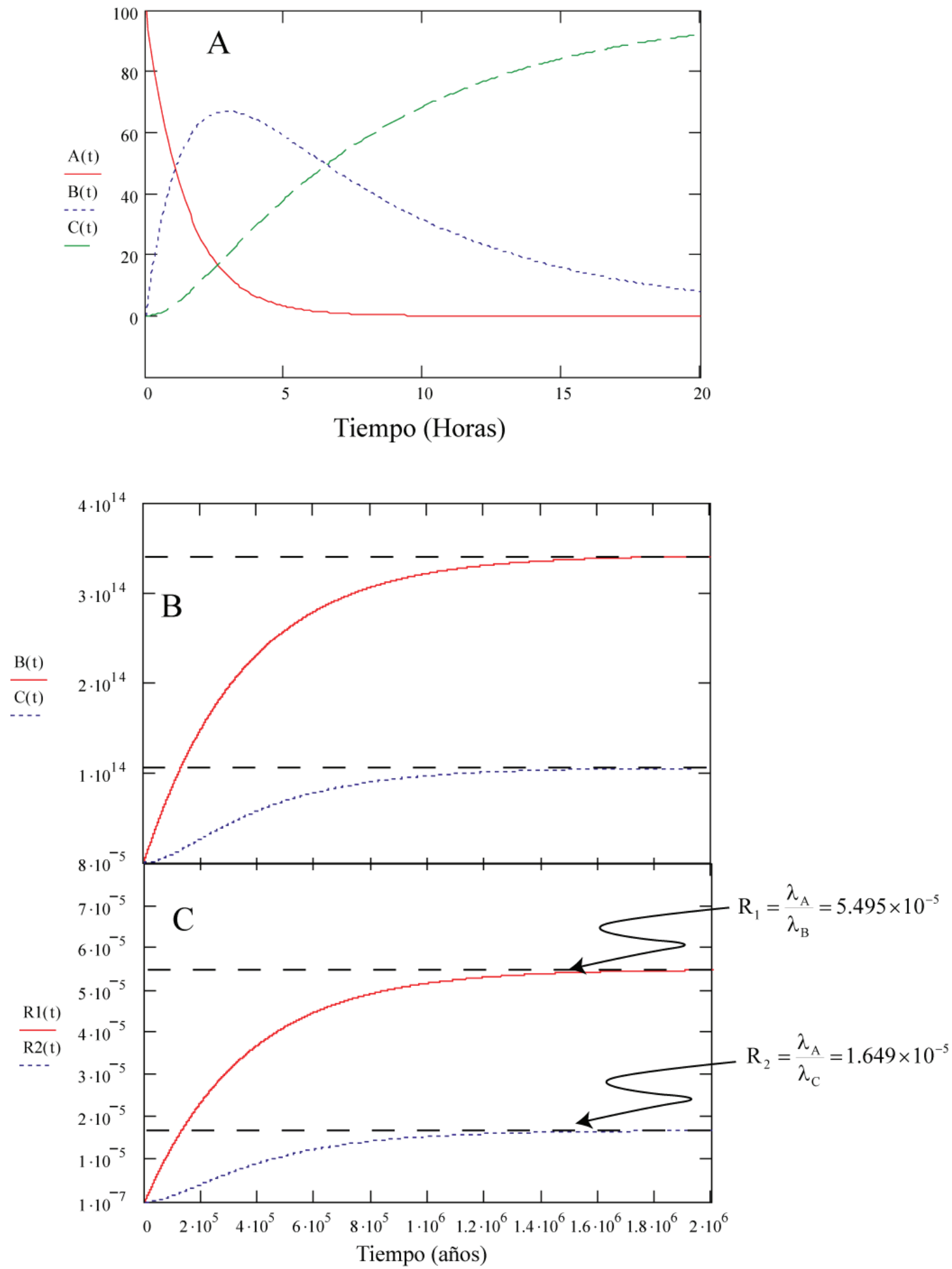

Figura 3. A) Abundancia de 3 isótopos que decaen bajo un esquema de cadena $(\mathrm{A} \rightarrow \mathrm{B} \rightarrow \mathrm{C})$ de acuerdo a las Ecuaciones 3,6 y 7 con vidas medias de 1 , 5 y 50 horas, respectivamente, en el caso hipotético en el que se inicia con 100 partículas del isótopo A. B) y C) Demostración gráfica de la Ecuación 8 , mostrando la abundancia absoluta y relativa, respectivamente, de los isótopos $\mathrm{B}$ y $\mathrm{C}$ al alcanzar el equilibrio secular, cuando sus vidas medias son iguales a las del ${ }^{238} \mathrm{U},{ }^{234} \mathrm{U}$ y ${ }^{230} \mathrm{Th}$.

sistemas están basados en determinar que tan cercana o lejana del equilibrio secular se encuentra cierta composición isotópica, es decir, a partir de mediciones de desequilibrio en las cadenas de decaimiento es posible establecer la fecha a partir de la cual se rompieron las condiciones de equilibrio secular, con la ventaja de que no es necesario saber la 
cantidad inicial del isótopo padre. La sistemática de estos sistemas se detalla ampliamente más abajo.

Una implicación directa de los sistemas de datación basados en el desequilibrio en las cadenas de decaimiento es que, a diferencia de otros geocronómetros radioactivos ( $p$. ej. Rb-Sr, Sm-Nd o U-Pb), hay un límite temporal superior por encima del cual no es posible realizar dataciones y el cual será directamente proporcional a la constante de decaimiento. De esta manera, los estudios enfocados a la disminución de la incertidumbre en la medición de la abundancia relativa de los isótopos, $\mathrm{N}_{\mathrm{B}} / \mathrm{N}_{\mathrm{A}}$, (p.e., Andersen et al., 2004; Potter et al., 2005a) no sólo tienen como objetivo obtener dataciones más precisas, sino también extender al máximo el rango de edades que es posible fechar isotópicamente.

\section{Principios y modelos para fechar sedimentos recientes con ${ }^{210} \mathrm{~Pb}$}

La técnica más utilizada para establecer un marco geocronológico en los sedimentos recientes lacustres y marinos ( 100 años), es la aplicación del radioisótopo natural ${ }^{210} \mathrm{~Pb}$ y el radioisótopo artificial ${ }^{137} \mathrm{Cs}$, debido a que sus vidas medias son alrededor de 22 y 30 años respectivamente (Goldberg y Koide, 1962; Krishnaswamy et al., 1971; Koide et al., 1972; Krishnaswamy y Lal, 1978; Robbins, 1978; Appleby y Oldfield, 1983; Oldfield y Appleby, 1985; Appleby, 2001; Carroll y Lerche, 2003). La medición de la actividad del ${ }^{210} \mathrm{~Pb}$ puede llevarse a cabo mediante espectrometría alfa de dilución isotópica a través de su nieto ${ }^{210} \mathrm{Po}$, o directamente mediante espectrometría gamma.

\subsection{Principios de la datación con ${ }^{210} \mathrm{~Pb}$}

Dentro de la serie de desintegración del ${ }^{238} \mathrm{U}$ se encuentra el ${ }^{226} \mathrm{Ra}$ (vida media $=1602$ años), que se desintegra para generar el gas ${ }^{222} \mathrm{Rn}$ (vida media= 3.83 días; Figura 4). Parte del ${ }^{222} \mathrm{Rn}$ generado en la parte externa de la litósfera se difunde a la atmósfera con una tasa constante de aproximadamente 42 átomos $\mathrm{min}^{-1} \mathrm{~cm}^{-2}$. Posteriormente se desintegra a través de varios productos de vida media muy corta hasta dar origen al ${ }^{210} \mathrm{~Pb}$. El ${ }^{210} \mathrm{~Pb}$ atmosférico (como sólido) regresa a la litosfera durante eventos de precipitación pluvial y/o adsorbido a aerosoles (Figura 5). Su tiempo de residencia atmosférica es de sólo 5 a 10 días (Krishnaswamy et al., 1971).

El método de datación de sedimentos con ${ }^{210} \mathrm{~Pb}$ se fundamenta en la determinación de la actividad del ${ }^{210} \mathrm{~Pb}$ atmosférico $\left({ }^{210} \mathrm{~Pb}\right.$ no soportado o en exceso) presente en los sedimentos marinos y lacustres. Éste debe ser distinguido $\mathrm{del}{ }^{210} \mathrm{~Pb}$ formado por la desintegración del ${ }^{222} \mathrm{Rn}$ que no logró escapar hacia la atmósfera y que se encuentra en equilibrio secular con su abuelo de vida larga, el ${ }^{226} \mathrm{Ra}$. Este ${ }^{210} \mathrm{~Pb}$ se asocia a los valores de fondo del sistema litosférico

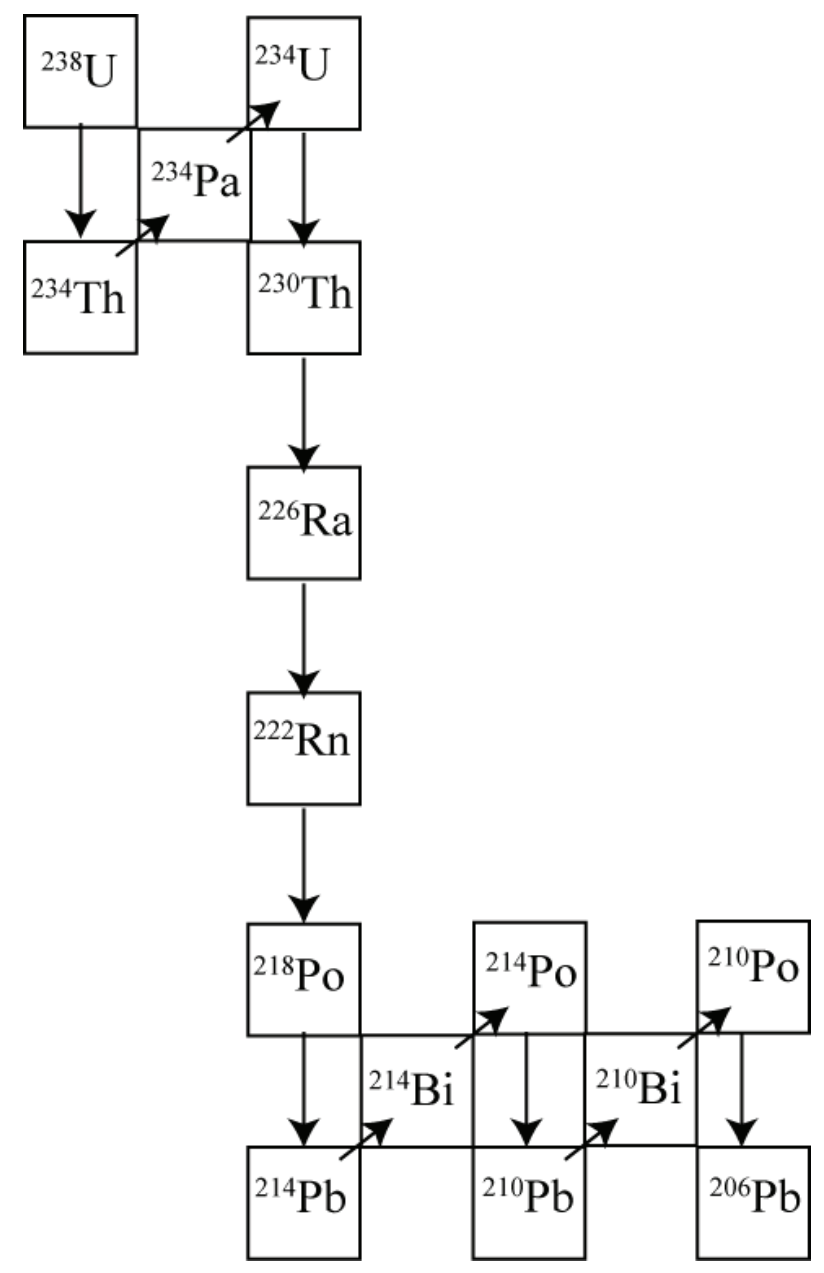

Figura 4. Cadena de decaimiento del ${ }^{238} \mathrm{U}$. Cada flecha vertical indica un decaimiento $\alpha$, mientras que las que apuntan hacia la esquina superior derecha son indicativas de un decaimiento $\beta+$.

y se denomina ${ }^{210} \mathrm{~Pb}$ soportado. Su actividad en el sedimento está controlada principalmente por la mineralogía del substrato y se considera constante a lo largo de una columna determinada de sedimento. La subsecuente incorporación del ${ }^{210} \mathrm{~Pb}$ atmosférico al sedimento provoca un 'exceso' de este isótopo en relación a los valores constantes del ${ }^{210} \mathrm{~Pb}$ soportados. A este exceso se le denomina ${ }^{210} \mathrm{~Pb}$ no soportado (en exceso) y se distingue del ${ }^{210} \mathrm{~Pb}$ soportado al restar la concentración del ${ }^{226} \mathrm{Ra}$ (en equilibrio con el ${ }^{210} \mathrm{~Pb}$ soportado) del ${ }^{210} \mathrm{~Pb}$ total:

$$
{ }^{210} \mathrm{~Pb}_{\text {Exceso }}={ }^{210} \mathrm{~Pb}_{\text {Total }}-{ }^{226} \mathrm{Ra}
$$

Ecuación 9

El fechado con ${ }^{210} \mathrm{~Pb}$ se establece a partir de los valores de la actividad del ${ }^{210} \mathrm{~Pb}$ no soportado, que está en función de la profundidad dentro del sedimento. Comúnmente se trata de las primeras decenas de centímetros, hasta más de un metro, dependiendo de las tasas de sedimentación. 


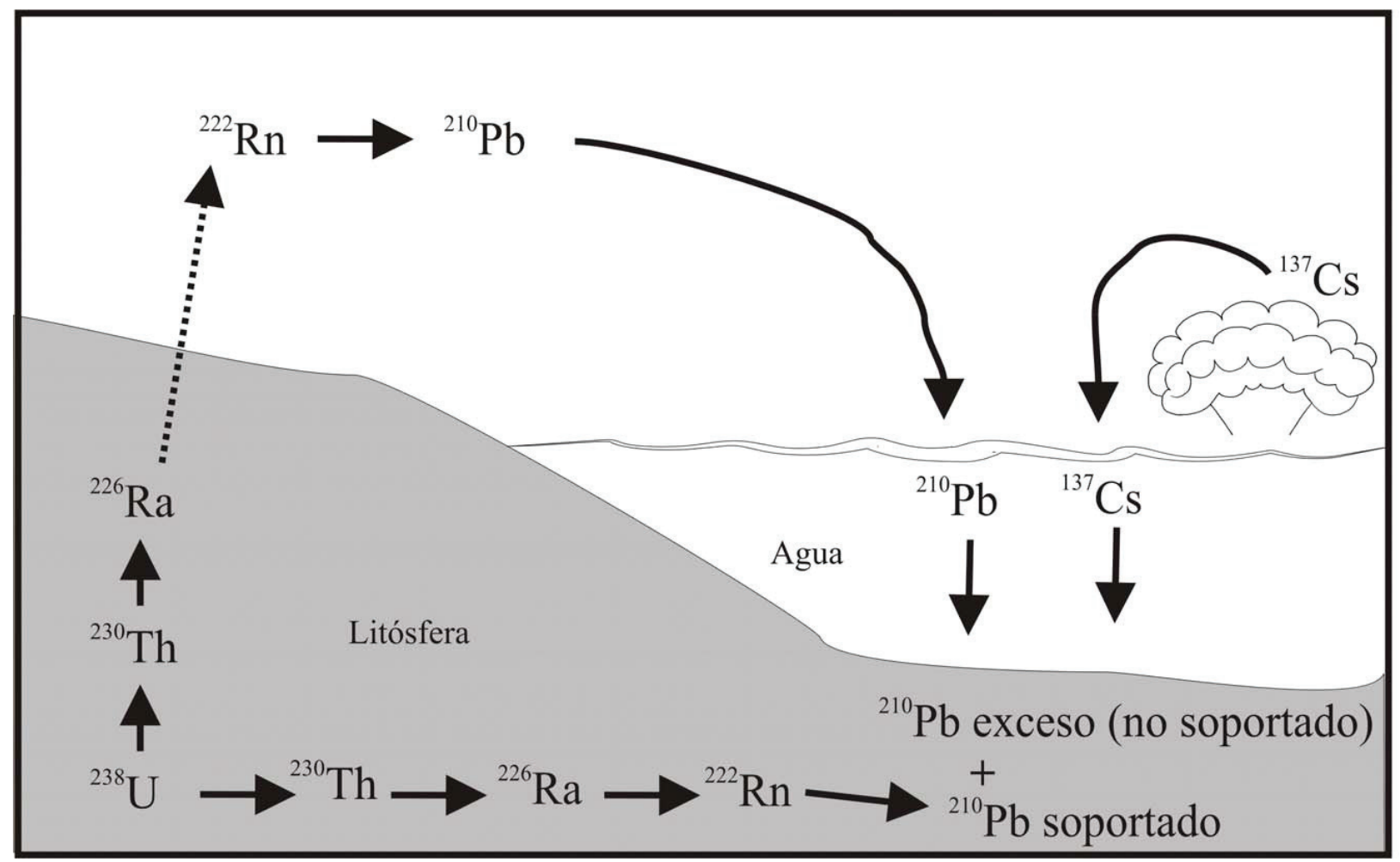

Figura 5. Esquema simplificado del decaimiento radiactivo del ${ }^{238} \mathrm{U}$ que muestra la acumulación en sedimentos subacuáticos de ${ }^{210} \mathrm{~Pb}$ exceso (no soportado) transportado por vía atmosférica y de ${ }^{210} \mathrm{~Pb}$ formado in situ (soportado), así como las explosiones y accidentes nucleares como fuente de ${ }^{137} \mathrm{Cs}$ hacia los sedimentos.

A diferencia del ${ }^{210} \mathrm{~Pb}$ soportado, la actividad del ${ }^{210} \mathrm{~Pb}$ no soportado presenta valores exponencialmente decrecientes con respecto a la profundidad, de acuerdo con su constante de decaimiento radioactivo (Figura 6). Las desviaciones de esta distribución exponencial nos permiten identificar las posibles alteraciones en el ambiente sedimentario y/o del flujo de material del sitio de estudio (Oldfield y Appleby, 1985).

Para la correcta aplicación del método de fechado con ${ }^{210} \mathrm{~Pb}_{\text {exceso }}$ debe considerarse que la actividad de ${ }^{210} \mathrm{~Pb}_{\text {exceso }}$ en la interfase agua-sedimento está en función de la tasa de flujo del mismo ${ }^{210} \mathrm{~Pb}_{\text {exceso }}$ y de la tasa de sedimentación. Por lo menos uno de estos factores debe ser conocido o asumido para transformar los datos de ${ }^{210} \mathrm{~Pb}$ exceso en edades. Otra de las consideraciones que se debe tener en mente es la posibilidad de migración o intercambio de ${ }^{210} \mathrm{~Pb}_{\text {exceso }}$ entre el agua intersticial y los sedimentos. Se ha demostrado que el ${ }^{210} \mathrm{~Pb}$ puede ser removilizado de la superficie de los sedimentos hacia las aguas intersticiales y que éstas, a su vez, pueden ser removilizadas hacia la columna de agua que sobreyace a los sedimentos (Santschi et al., 1983; Benoit y Hemod, 1991).

Debido a los procesos anteriormente descritos, se ha optado por desarrollar modelos que se ajusten a las diferentes condiciones ambientales (flujos de ${ }^{210} \mathrm{~Pb}$, sedimentación y removilización).

\subsection{Modelos de depósito de ${ }^{210} \mathrm{~Pb}$}

Bajo algunos términos, la edad de depósito de los sedimentos puede ser estimada en perfiles verticales de sedimentos (Appleby y Oldfield, 1983; Appleby, 2001; Marques et al., 2006) a partir de un modelo de decaimiento simple:

$$
{ }^{210} \mathrm{~Pb}_{\mathrm{z}}={ }^{210} \mathrm{~Pb}_{0} \mathrm{e}^{-\lambda \mathrm{t}}
$$

Ecuación 10

Donde:

${ }^{210} \mathrm{~Pb}_{\mathrm{z}}=$ la actividad de ${ }^{210} \mathrm{~Pb}$ a una profundidad $\mathrm{z}$.

${ }^{210} \mathrm{~Pb}_{0}=$ la actividad de ${ }^{210} \mathrm{~Pb}$ en la superficie.

$\lambda=$ la constante de decaimiento de ${ }^{210} \mathrm{~Pb}\left(0.0311 \mathrm{a}^{-1}\right)$.

$\mathrm{t}=$ el tiempo que ha pasado desde que se produjo la sedimentación hasta que este sedimento alcanza una profundidad $\mathrm{z}$.

El perfil de actividad de ${ }^{210} \mathrm{~Pb}_{\text {exceso }}$ en sedimentos de $\sim 100$ años, permite calcular la edad del sedimento utilizando principalmente los siguientes modelos: a) el modelo CFCS (flujo constante/sedimentación constante, por sus siglas en inglés), b) el modelo CIC (concentración inicial constante) y el modelo CRS (tasa de suministro constante, por sus siglas en inglés).

Estos modelos presuponen las siguientes premisas: 


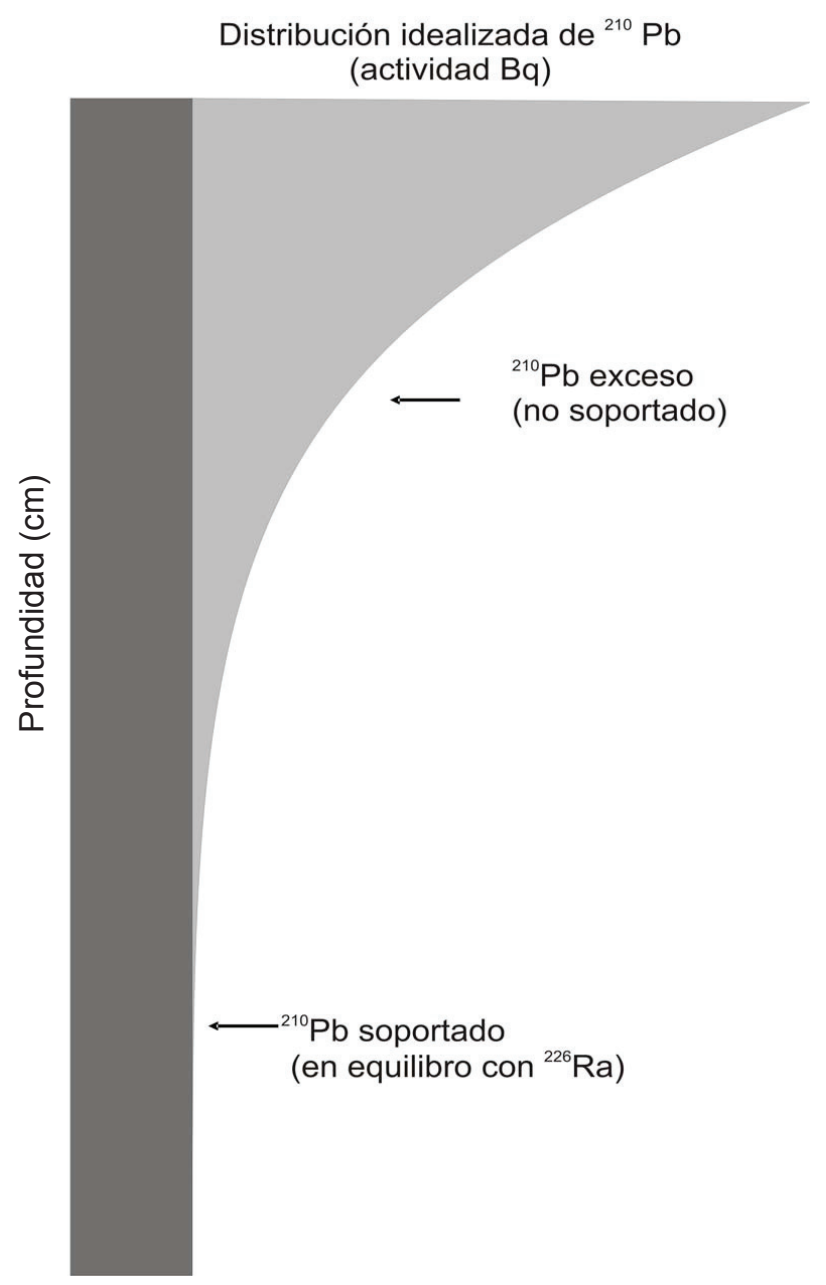

Figura 6. Distribución idealizada del ${ }^{210} \mathrm{~Pb}$ en sedimentos subacuáticos, de donde se calcula la edad a diferentes profundidades $\left(t=\ln \left(\mathrm{A}_{0} /\right.\right.$ $\left.\mathrm{A}_{\mathrm{x}} / \lambda\right)$ utilizando la constante de decaimiento radiactivo para el ${ }^{210} \mathrm{~Pb}$ $(\lambda=0.03114)$, la actividad inicial ( $\mathrm{A}_{0}$ en superficie) y la actividad a diferentes profundidades $\left(\mathrm{A}_{\mathrm{x}}\right)$.

1. Existe un flujo constante de ${ }^{210} \mathrm{~Pb}$ atmosférico hacia el sistema.

2. El ${ }^{210} \mathrm{~Pb}$ atmosférico no presenta movilidad postdepositacional dentro del sedimento.

3. No existe pérdida de ${ }^{210} \mathrm{~Pb}$ desde el sedimento hacia el agua de un sistema subacuático. Los sedimentos son considerados como un sistema cerrado.

4.La concentración de ${ }^{210} \mathrm{~Pb}$ disuelto en las aguas de sistemas lacustres es prácticamente cero.

5. El ${ }^{210} \mathrm{~Pb}$ tiene un tiempo de residencia atmosférico relativamente corto en relación al tiempo de residencia de un sistema acuático, de tal forma que el ${ }^{210} \mathrm{~Pb}$ atmosférico que alcanza la columna de agua es transferido rápidamente al sedimento.

\subsubsection{Modelo CFCS}

El modelo de flujo constante y tasa de sedimentación constante, supone que el flujo de ${ }^{210} \mathrm{~Pb}_{\text {exceso }}$ es constante y que la tasa de sedimentación de masa (de sedimento seco) es también constante. Representado en un diagrama semilogarítmico la evolución de ${ }^{210} \mathrm{~Pb}_{\text {exc }}$ con la profundidad se convierte en una línea y su pendiente permite calcular la tasa de sedimentación.

$$
\mathrm{A}_{\text {mTOT }}=\mathrm{A}_{0 \text { (exceso) }} \mathrm{e}^{-\lambda \mathrm{m} / \mathrm{r}}+\mathrm{A}_{\text {soportado }}
$$

\section{Ecuación 11}

Donde:

$\mathrm{A}_{\text {mTOT }}=$ actividad de ${ }^{210} \mathrm{~Pb}$ total $\left(\mathrm{mBqg}^{-1}\right.$ en masa de sedimento seco).

$\mathrm{A}_{0 \text { (exceso) }}=$ actividad inicial de ${ }^{210} \mathrm{~Pb}$ en la interfase agua/ sedimento ( $\mathrm{mBq} \mathrm{g}^{-1}$ sedimento seco).

$\mathrm{m}=$ masa (sedimento seco) acumulativa $\left(\mathrm{gcm}^{-2}\right)$ a una profundidad dada.

$\lambda=$ constante de decaimiento del ${ }^{210} \mathrm{~Pb}\left(0.03114 \mathrm{a}^{-1}\right)$.

$\mathrm{r}=$ tasa de acumulación de masa (sedimento seco, $\mathrm{g}$ $\left.\mathrm{cm}^{-2} \mathrm{a}^{-1}\right)$.

$\mathrm{A}_{\text {(soportado) }}=$ actividad de ${ }^{210} \mathrm{~Pb}$ soportado $\left(\mathrm{mBq} \mathrm{g}^{-1}\right)$.

\subsubsection{Modelo CIC}

El modelo de Concentración Inicial Constante, CIC, supone una concentración inicial constante y tasa de sedimentación de masa de sedimento variable. Este modelo requiere que todas las partículas de sedimento tengan la misma actividad inicial de ${ }^{210} \mathrm{~Pb}_{\text {exceso }}$. Así, la actividad inicial de ${ }^{210} \mathrm{~Pb}$ exceso por gramo de sedimento seco no ha cambiado con respecto a su depósito en el sitio de toma del núcleo (Krishnaswamy y Lal, 1978; Robbins, 1978). La concentración de ${ }^{210} \mathrm{~Pb}_{\text {exceso }}$ en la parte superior del registro sedimentario (donde no hay mezcla) es constante a través del tiempo, debido a que el ${ }^{210} \mathrm{~Pb}_{\text {exceso }}$ se integra con los sedimentos, e inversamente proporcional al flujo particulado (Robbins y Edgington, 1975).

Este modelo se considera útil solamente para condiciones no variables como, por ejemplo, el mar profundo, en donde existe un exceso del reservorio de ${ }^{210} \mathrm{~Pb}_{\text {exceso }}$ en la columna de agua a través del tiempo y la cantidad de ${ }^{210} \mathrm{~Pb}$ incorporado a las partículas es constante. Estas características ambientales permiten el secuestro constante de ${ }^{210} \mathrm{~Pb}$. Asimismo, este modelo considera, que la edad del sedimento en cada capa puede ser calculada aplicando la siguiente ecuación.

$$
\mathrm{t}=\frac{1}{\lambda} \times \frac{\mathrm{C}_{(0)}}{\mathrm{C}}
$$

Ecuación 12

Donde:

$\mathrm{C}_{(0)}=$ actividad de ${ }^{210} \mathrm{~Pb}_{\text {exceso }}$ en la superficie.

$\mathrm{C}=$ actividad de ${ }^{210} \mathrm{~Pb}_{\text {exceso }}$ en la muestra a ser datada.

$\lambda=$ constante de decaimiento de ${ }^{210} \mathrm{~Pb}_{\text {exceso }}=0.03114 \mathrm{a}^{-1}$

$\mathrm{t}=$ edad del sedimento. 


\subsubsection{Modelo CRS}

El modelo de tasa de suministro constante fue originalmente propuesto por Goldberg y Koide (1962) y desarrollado con más detalle por Appleby y Oldfield (1978). En este modelo se considera que la tasa de flujo absoluto $\mathrm{de}^{210} \mathrm{~Pb}_{\text {exceso }}$ en la interfase agua-sedimento permanece constante, independientemente de la tasa de sedimentación. Se considera que la actividad inicial de ${ }^{210} \mathrm{~Pb}_{\text {exceso }}$, es inversamente proporcional a la tasa de sedimentación, de tal manera que a altas tasas de sedimentación se puede tener una baja concentración de ${ }^{210} \mathrm{~Pb}_{\text {exceso }}$. Este modelo supone que la edad del sedimento puede ser calculada aplicando la siguiente ecuación.

$$
\mathrm{t}=\frac{1}{\lambda} \times \frac{\mathrm{A}_{(0)}}{\mathrm{A}}
$$

Ecuación 13

\section{Donde:}

$\mathrm{A}_{(0)}=$ inventario de la actividad ${ }^{210} \mathrm{~Pb}_{\text {exceso }}$ en superficie.

$\mathrm{A}=$ inventario de ${ }^{210} \mathrm{~Pb}_{\mathrm{xs}}$ en todos los sedimentos debajo de la muestra a datar.

$\lambda=$ constante de decaimiento de ${ }^{210} \mathrm{~Pb}_{\text {exceso }}=0.03114 \mathrm{a}^{-1}$.

$\mathrm{t}=$ edad del sedimento.

Debido a las discrepancias en la datación con ${ }^{210} \mathrm{~Pb}_{\text {exceso }}$ reportadas en múltiples artículos, Smith (2001) menciona: "La geocronología con ${ }^{210} \mathrm{~Pb}_{\text {excess }}$ se debe validar utilizando por lo menos un trazador independiente que proporcione por separado la edad de un horizonte estratigráfico inequívoco". Para establecer una cronología válida, aun cuando se utilicen otros marcadores cronoestratigráficos como apoyo a la datación con ${ }^{210} \mathrm{~Pb}_{\text {exceso }}$, se recomienda ampliamente conocer la dinámica de depósito del área de estudio, así como la aparición de diversos marcadores cronoestratigráficos de origen antropogénico. En este sentido, Carrol y Lerche (2003) mencionan que con frecuencia se pueden asignar edades erróneas al no considerar los supuestos mencionados anteriormente.

Uno de estos trazadores adicionales al ${ }^{210} \mathrm{~Pb}$ puede ser el ${ }^{137} \mathrm{Cs}$ o radiocesio. Este radioisótopo tiene una vida media de 30.3 años y es producido por las reacciones termonucleares, principalmente las bombas nucleares a mediados del siglo $\mathrm{XX}$, por lo que la presencia de este isótopo en sistemas naturales está directamente relacionada a la actividad termonuclear atmosférica (Figura 5). El ${ }^{137} \mathrm{Cs}$ es adsorbido y fijado rápidamente por las partículas en los sedimentos, especialmente en los minerales de arcilla y en particular la vermiculita (Staunton, 2002). El depósito del ${ }^{137} \mathrm{Cs}$ en el hemisferio norte inició en 1952, con un pico máximo en 1963 y 1964. El accidente de Chernobyl, ocurrido en 1986, produjo un segundo pulso en el ${ }^{137} \mathrm{Cs}$ atmosférico el cual es detectable en muestras ambientales (Pourchet et al., 1986, 1988). Varios autores han estudiado el depósito de ${ }^{137} \mathrm{Cs}$ en diversos sistemas lacustres (Pennington et al., 1973; Robbins y Edgington, 1975; Robbins, 1978). Al igual que el ${ }^{210} \mathrm{~Pb}$ exceso, la precipitación del ${ }^{137} \mathrm{Cs}$ ha servido como un trazador muy valioso en sedimentos en varios casos de estudios ambientales, especialmente los que proporcionan información de las tasas de erosión de suelos o las tasas de sedimentación en ambientes lacustres.

\section{Datación a partir del desequilibrio en la cadena ${ }^{238} \mathbf{U}^{234} \mathrm{U}^{230} \mathrm{Th}$}

La cadena de decaimiento del ${ }^{238} \mathrm{U}$ se detalla en la Figura $3 \mathrm{y}$, de manera simplificada, se expresa de la siguiente manera:

$\begin{array}{ccc} & \text { Vida Media (años) } & \text { Referencia } \\ { }^{238} \mathrm{U} \rightarrow{ }^{234} \mathrm{U}+\alpha & (4.4383 \pm 0.0024) \times 10^{9} & \text { Jaffey } \text { et al., } 1971 \\ { }^{234} \mathrm{U} \rightarrow{ }^{230} \mathrm{Th}+\alpha & 245250 \pm 490 & \text { Cheng } \text { et al.,2000b } \\ { }^{230} \mathrm{Th} \rightarrow{ }^{226} \mathrm{Ra}+\alpha & 75690 \pm 230 & \text { Cheng } \text { et al.,2000b }\end{array}$

donde $\alpha$ equivale a una partícula alfa, es decir ${ }^{4} \mathrm{He}^{2+}$. Aunque estrictamente el ${ }^{238} \mathrm{U}$ decae de acuerdo a la siguiente reacción:

$$
\begin{gathered}
{ }^{238} U \rightarrow{ }^{234} \mathrm{Th}+\alpha \\
\text { en general se considera que } \\
{ }^{238} \mathrm{U} \rightarrow{ }^{234} \mathrm{U}+\alpha+2 \beta^{+}
\end{gathered}
$$

donde $\beta^{+}$equivale a la partícula beta positiva, o positrón. Debido a que las transformaciones

$$
\begin{aligned}
{ }^{234} \mathrm{Th} & \rightarrow{ }^{234} \mathrm{~Pa}+\beta^{+} \\
& \mathrm{y} \\
{ }^{234} \mathrm{~Pa} & \rightarrow{ }^{234} \mathrm{U}+\beta^{+},
\end{aligned}
$$

tienen vidas medias de 24.1 días y 6.69 horas, respectivamente (Audi et al., 1997), una vez formados, alcanzan el equilibrio secular con el isótopo padre $\left({ }^{238} \mathrm{U}\right.$ y ${ }^{234} \mathrm{Th}$, respectivamente) rápidamente. La aplicación de las Ecuaciones 3, 6 y 7 al sistema ${ }^{238} \mathrm{U} \rightarrow{ }^{234} \mathrm{U} \rightarrow{ }^{230} \mathrm{Th}$, genera para ${ }^{234} \mathrm{U} /{ }^{238} \mathrm{U}$ :

$$
\begin{gathered}
{\left[\frac{{ }^{234} \mathrm{U}}{{ }^{238} \mathrm{U}}\right]=\left(\left[\frac{{ }^{234} \mathrm{U}}{{ }^{238} \mathrm{U}}\right]_{0}-1\right) \times \mathrm{e}^{-\lambda_{234} \mathrm{t}}} \\
\delta^{234} \mathrm{U}=\delta^{\mathrm{O} 34} \mathrm{U}_{0} \mathrm{e}^{-\lambda_{234} \mathrm{t}}
\end{gathered}
$$

Ecuación 14

donde los corchetes indican actividad o relación de actividades, el subíndice cero denota la relación de actividades inicial y $\delta^{234} U=\left(\left[{ }^{234} U / 238 U\right]-1\right) \times 1000$. Aunque se puede proponer una ecuación similar para el sistema ${ }^{238} \mathrm{U} \rightarrow{ }^{230} \mathrm{Th}$, hay una serie de procesos geoquímicos (discutidos en más adelante) que afectan la relación ${ }^{234} \mathrm{U} /{ }^{238} \mathrm{U}$ $\mathrm{y}$, por lo tanto, la cadena de decaimiento, por lo que dicha simplificación sólo es aplicable a aquellos casos donde no haya fraccionamiento isotópico entre ${ }^{234} \mathrm{U} /{ }^{238} \mathrm{U}$. Como consecuencia de la estrecha interrelación entre las tres principales cadenas de decaimiento, las ecuaciones 3,6 y 7 , no son independientes y forman parte de un sistema de 
ecuaciones diferenciales, cuya solución es (Broecker, 1963):

$$
\begin{gathered}
{\left[\frac{{ }^{230} \mathrm{Th}}{{ }^{238} \mathrm{U}}\right]=1-\mathrm{e}^{-\lambda_{230} \mathrm{t}}+\frac{\lambda_{230}}{\lambda_{230}-\lambda_{234}} \times\left[\left[\frac{{ }^{234} \mathrm{U}}{{ }^{238} \mathrm{U}}\right]-1\right] \times\left(1-\mathrm{e}^{\left(\lambda_{234}-\lambda_{230}\right) \mathrm{t}}\right)} \\
{\left[\frac{\mathrm{o} \mathrm{Th}}{{ }^{238} \mathrm{U}}\right]=1-\mathrm{e}^{-\lambda_{230} \mathrm{t}}+\frac{\lambda_{230}}{\lambda_{230}-\lambda_{234}} \times\left(\frac{\delta^{234} \mathrm{U}}{1000}\right) \times\left(1-\mathrm{e}^{-\left(\lambda_{234}-\lambda_{230}\right) \mathrm{t}}\right)}
\end{gathered}
$$

Ecuación 15

La Ecuación 15 puede resolverse de manera iterativa para $t$, y calcular la edad a partir de las relaciones de actividad modernas de $\left[{ }^{234} \mathrm{U} /{ }^{238} \mathrm{U}\right]$ o $\left(\delta^{234} \mathrm{U}\right)$ y $\left[{ }^{230} \mathrm{Th} /{ }^{238} \mathrm{U}\right]$, correspondiendo a la edad del último evento de fraccionamiento entre ${ }^{230} \mathrm{Th}$ y ${ }^{238} \mathrm{U}$. La Figura 7 muestra la solución gráfica de la Ecuación 15, y demuestra que se pueden trazar isócronas para un conjunto diferente de $\left[{ }^{234} \mathrm{U} /{ }^{238} \mathrm{U}\right]$ y $\left[{ }^{230} \mathrm{Th} /{ }^{238} \mathrm{U}\right]$, por lo que es necesario contar con un conocimiento de la evolución isotópica de cada sistema, en particular $\left[{ }^{234} \mathrm{U} /{ }^{238} \mathrm{U}\right]$, que indiquen la evolución isotópica en condiciones de sistema cerrado, antes de establecer conclusiones geocronológicas.

Las ecuaciones hasta aquí descritas asumen que todo el ${ }^{230} \mathrm{Th}$ se formó de manera radiogénica in situ, es decir, que en el momento en el cual cristalizó la fase mineral que capturó al U, está no incorporó ${ }^{230} \mathrm{Th}$ del medio. Lo anterior es aparentemente razonable en sistemas de baja temperatura, donde la diferencia de solubilidades entre el Th y el U permite suponer que el primero es prácticamente inmóvil (ver abajo). Desafortunadamente, lo anterior no siempre se cumple, ya que la incorporación de material detrítico (materia orgánica, arcillas) en las fases minerales susceptibles a ser datadas por series de U, acarrean consigo cantidades considerables de Th. De esta manera es necesario contar con alguna metodología que permita discernir entre el ${ }^{230} \mathrm{Th}$ formado radiogénicamente in situ, de aquel que fue incorporado al mineral junto con el material detrítico, lo cual se logra utilizando al ${ }^{232} \mathrm{Th}$ como indicador de la presencia de contribuciones alogénicas.

El ${ }^{232} \mathrm{Th}$ es el único isótopo no radiogénico del Th, $\mathrm{y}$ no está relacionado con la cadena de decaimiento del ${ }^{238} \mathrm{U}$. Debido a su baja solubilidad y alta afinidad al material particulado, es comúnmente asociado a la presencia de fases minerales no solubles. De esta manera, una alta concentración de ${ }^{232} \mathrm{Th}$ en la fase mineral a datar indica, a grandes rasgos, que las condiciones geoquímicas para la movilización del Th, indistintamente del isótopo, estuvieron presentes en el momento de formación del mineral a datar y, por lo tanto, es altamente factible que una fracción del ${ }^{230} \mathrm{Th}$ presente en la fase mineral no se haya formado in-situ. Aunque se han realizado diversos

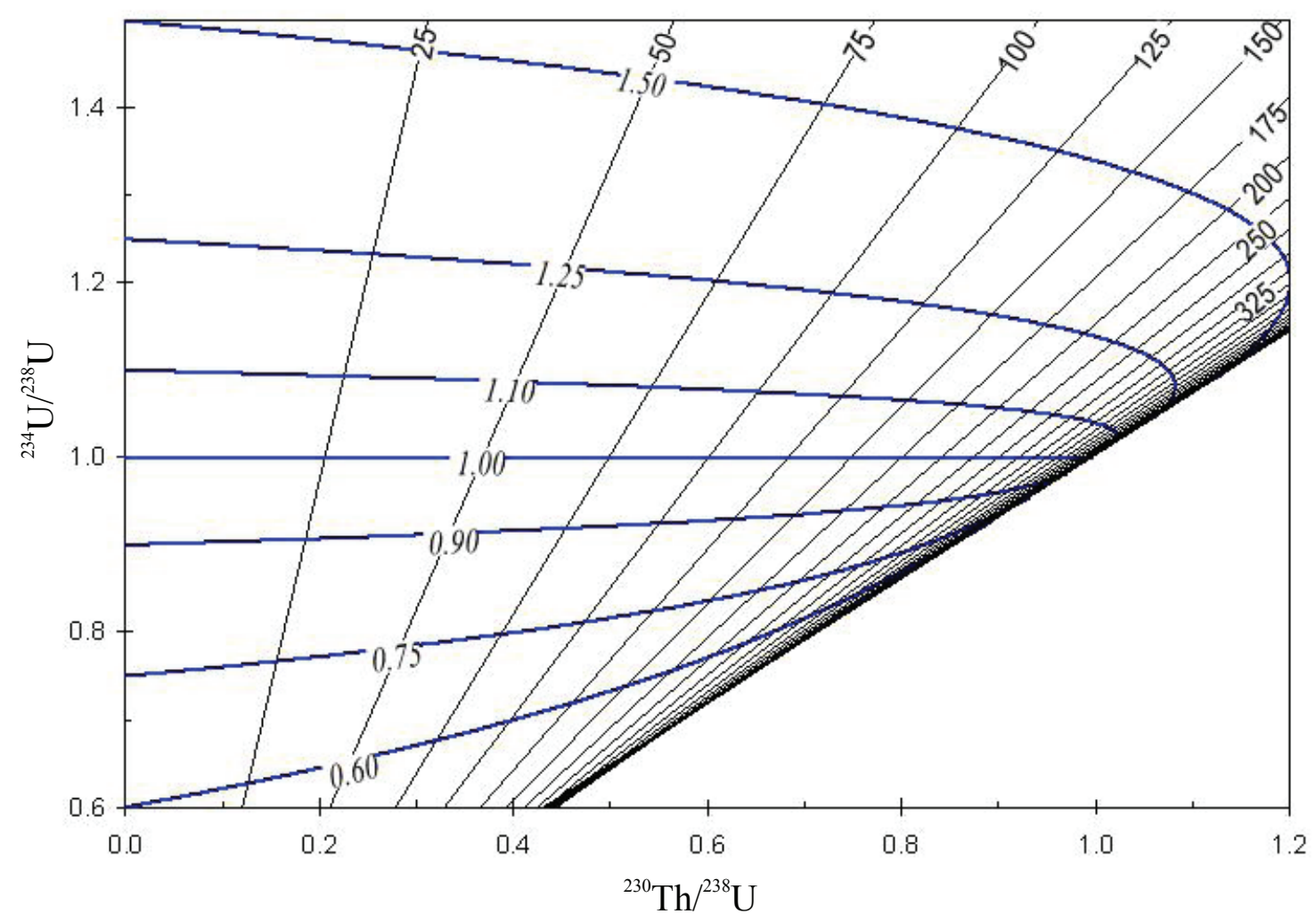

Figura 7. Solución gráfica de la Ecuación 15 que muestra las diferentes isocronas (líneas verticales a semi-verticales), así como algunas líneas de evolución $\mathrm{de}^{234} \mathrm{U} /{ }^{238} \mathrm{U}$ (líneas azules) en función del tiempo o ${ }^{230} \mathrm{Th} /{ }^{238} \mathrm{U}$. 
esfuerzos por disolver, preferentemente, la fase no detrítica (Schwarcz y Latham, 1989), se ha demostrado que dichos métodos no pueden evitar disolver una fracción de los núclidos presentes en la fase detrítica (Kaufman, 1993; Alcaraz Pelegrina y Martínez-Aguirre, 2005) resultando en composiciones isotópicas difíciles de interpretar. Cuando la relación ${ }^{238} \mathrm{U} /{ }^{232} \mathrm{Th}$ en la muestra es relativamente alta, es posible hacer correcciones matemáticas basadas en el hecho de que la fase analizada es el resultado de una mezcla entre un componente autigénico y otro alóctono. Dichas correcciones utilizan al ${ }^{232} \mathrm{Th}$ como indicador de material alóctono (Bischoff y Fitzpatrick, 1991; Luo y Ku, 1991), pero carecen de un cálculo detallado de las incertidumbres asociadas a la corrección. Dicho problema es resuelto por Ludwig y Titterington (1994), quienes utilizan regresiones lineales robustas ponderadas por la incertidumbre de las mediciones, así como métodos estadísticos de máxima probabilidad. Estos métodos son explicados con mayor detalle en Ludwig (2003). En general consisten en calcular ${ }^{230} \mathrm{Th} /{ }^{232} \mathrm{Th}$ de la fracción detrítica y su grado de contribución a la composición de la muestra analizada, con el fin de poder sustraer la aportación de ${ }^{230} \mathrm{Th}$ detrítico al ${ }^{230} \mathrm{Th}$ total.

\subsection{Procesos que alteran el equilibrio secular}

Los procesos que dan origen al fraccionamiento de los isótopos pertenecientes a la cadena de decaimiento del ${ }^{238} \mathrm{U}$ se pueden diferenciar entre aquellos basados en las diferentes propiedades químicas de los isótopos durante procesos de diferenciación geoquímica, principalmente el intemperismo y meteorización, y aquellos producto del decaimiento radioactivo. El U es un elemento litófilo que se encuentra en la naturaleza en dos estados de oxidación, $4+$ y $6+$. $\mathrm{El} \mathrm{U}^{4+}$ es un ácido duro y con alto potencial iónico (Bernal y Railsback, 2008), por lo que es poco soluble en contacto con disoluciones acuosas a temperatura ambiente y bajo condiciones reductoras (Langmuir, 1978). De acuerdo a sus propiedades atómicas, comúnmente se encuentra enriquecido en suelos y fases residuales de intemperismo (Bernal y Railsback, 2008), ya sea formando óxidos simples, o asociado a minerales como circón, apatito o xenotimo. Bajo condiciones oxidantes el $\mathrm{U}$ adquiere el estado de oxidación $6+$ que, debido a su alto potencial iónico y alta dureza, forma el oxi-catión $\mathrm{UO}_{2}{ }^{2+}$, el cual es altamente soluble. Por otra parte, el Th sólo se encuentra como $\mathrm{Th}^{4+}$ que, de manera similar al $\mathrm{U}^{4+}$, es extremadamente insoluble debido a que forma óxidos simples de muy baja solubilidad (Langmuir y Herman, 1980) y tiene un alto grado de compatibilidad con fases minerales resistentes a procesos de intemperismo.

La marcada diferencia en solubilidad entre el $\mathrm{UO}_{2}{ }^{2+}$ y el $\mathrm{Th}^{4+}$ tiene como consecuencia que, durante procesos de diferenciación geoquímica por disolución a baja temperatura, por ejemplo el intemperismo químico, el U sea fácilmente removido de la fase sólida, mientras que el Th permanecerá en las fases minerales residuales. El U se mantendrá en solución hasta que cambien las condiciones fisicoquímicas de ésta, y permita la precipitación de alguna fase mineral (calcita, oxi-hidróxidos de Fe, ópalo, etc.) que incorpore al $U$ de la solución dentro de su estructura cristalina. La separación de $\mathrm{U}$ y Th por el proceso arriba descrito tiene como consecuencia que tanto el ${ }^{238} \mathrm{U}$ como el ${ }^{234} \mathrm{U}$ sean fácilmente solubilizados y movilizados, mientras que el ${ }^{230} \mathrm{Th}$ permanecerá inmóvil en el residuo. De esta manera, la fase mineral que incorporó al U puede ser fechada, en un principio, al cuantificar el ${ }^{230} \mathrm{Th}$ acumulado durante varios cientos o miles de años.

A pesar de que la diferencia en solubilidad entre $\mathrm{U}^{6+} \mathrm{y}$ $\mathrm{Th}^{4+}$ es altamente efectiva para generar un fraccionamiento significativo entre $\mathrm{Th}$ y $\mathrm{U}$, no discrimina per se entre los distintos isótopos de $\mathrm{U}$, por lo que este proceso no puede explicar el desequilibrio isotópico observado en diversos ambientes superficiales como suelos, agua y rocas intemperizadas (Ivanovich y Harmon, 1992). Inicialmente reportados por Cherdynstev (Cherdynstsev, 1955), enriquecimientos de ${ }^{234} \mathrm{U}$ relativos a ${ }^{238} \mathrm{U}$ son comunes en distintos ambientes superficiales. El papel del agua en dicho enriquecimiento parece ser fundamental, pues análisis de muestras superficiales provenientes de la Luna no muestran desequilibrio entre ${ }^{234} \mathrm{U}$ y ${ }^{238} \mathrm{U}$ (Rosholt y Tatsumoto, 1970, 1971). Para explicar tal observación, se han propuesto dos mecanismos no excluyentes, basados en la conservación de la cantidad de movimiento durante la emisión de partículas $\alpha$ por el decaimiento del ${ }^{238} \mathrm{U}$ :

1) Basado en el estudio de la disolución de isótopos de U en circones, Kigoshi (1971) propuso que el exceso de ${ }^{234} \mathrm{U}$ en aguas subterráneas puede ser explicado por la expulsión de ${ }^{234}$ Th por retro-impacto $\alpha$ (" $\alpha$-recoill") durante los eventos de decaimiento que suceden en un espacio de $55 \mathrm{~nm}$ de la superficie del cristal (Figura 8A). El ${ }^{234} \mathrm{Th}$ expulsado, decaerá rápidamente para formar ${ }^{234} \mathrm{U}$, el cual puede ser movilizado fácilmente por el agua.

2) Un mecanismo alternativo fue propuesto por Fleischer (1980), quien implantó átomos ${ }^{235} \mathrm{U}$ en la superficie de minerales al exponerlos a una película de ${ }^{239} \mathrm{PuO}_{2}\left({ }^{239} \mathrm{Pu}\right.$ $\left.\rightarrow{ }^{235} U+\alpha\right)$. Los especimenes con ${ }^{235} U$ implantado fueron expuestos a diferentes soluciones que podrían disolver el ${ }^{235} \mathrm{U}$. El análisis posterior de la película mineral mostró que la gran mayoría de los isótopos implantados fueron liberados a la solución. Trabajos posteriores sobre la implantación de ${ }^{235} \mathrm{U}$ sobre la superficie de distintos minerales demostraron que ésta genera daños en la estructura cristalina de los minerales donde se lleva a cabo, y que el isótopo implantado puede ser removido fácilmente por disolución acuosa (Fleischer, 1982), demostrando que los isótopos formados por decaimiento a localizados al final de una traza de fisión son removidos más fácilmente que aquellos que se encuentran en el interior de la estructura cristalina.

\subsection{Aplicaciones y limitaciones}

Los materiales que son más comúnmente fechados 
utilizando series de U son los carbonatos secundarios (estalagmitas, caliche, carbonatos pedogénicos, travertino) y biogénicos (corales) del cuaternario, debido a que en el ambiente en donde se forman el fraccionamiento entre U y Th es muy eficiente. De esta manera la datación de estalagmitas por series de $U$ ha permitido el establecimiento de cronologías robustas y de alta precisión para series de tiempo climáticas de alta resolución (Dorale et al., 2004). Entre estas, cabe mencionar por su relevancia los archivos climáticos de norte América durante los últimos 4 periodos interglaciales a partir de la composición de isótopos de oxígeno de un espeleotema subacuático (Ludwig et al., 1992; Winograd et al., 1992, 1997, 2006), así como el archivo de variaciones de la intensidad del monzón asiático durante los últimos 200000 años (Wang et al., 2001, 2005, 2006). Por otra parte, la datación por series de U de caliche, travertino y otros carbonatos secundarios (Rosholt, 1976; Szabo y O'Malley, 1985; Eikenberg et al., 2001) ha permitido establecer cronologías para la evolución geomorfológica de terrazas fluviales (Ludwig y Paces, 2002; Sharp et al., 2003; Fletcher et al., 2010).

Una de las primeras contribuciones significativas de los métodos geocronológicos basados en el desequilibrio en las series de $\mathrm{U}$ es la datación de fósiles de corales, ya que ha permitido establecer, con gran precisión, la magnitud y edad de las distintas trasgresiones marinas ocurridas durante los diferentes periodos glaciales e interglaciales durante los últimos 300000 años (Stirling et al., 1995, 2001; Esat et al., 1999; McCulloch et al., 1999; Cheng et al., 2000a; Yokoyama et al., 2000, 2001; Gallup et al., 2002; Lambeck et al., 2002; Cobb et al., 2003), establecer los cambios climáticos asociados (Ayling et al., 2006) e, incluso, establecer un marco geocronológico a los procesos de subsidencia de la corteza terrestre en Hawaii (Ludwig et al., 1991). Asimismo, la datación de material coralino ha permitido establecer cronologías independientes que,

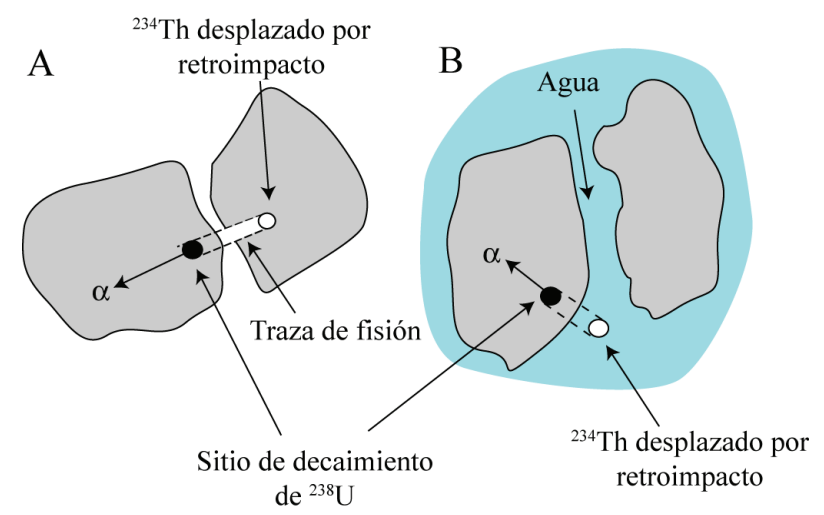

Figura 8. Mecanismos de fraccionamiento entre ${ }^{234} \mathrm{U}$ y ${ }^{238} \mathrm{U}$ basados en el retroimpacto- $\alpha$ ( $\alpha$-recoil) que influyen en la interacción de $U$ con agua. Estos modelos no son mutuamente excluyentes. A) modelo de Kigoshi (1971). B) Modelo de Fleischer (1980). en conjunto con otros métodos de datación, han permitido la calibración de las dataciones basadas en ${ }^{14} \mathrm{C}$, como se describe arriba (Reimer et al., 2004).

La gran cantidad de aplicaciones de la datación de corales por desequilibrio en las series de ${ }^{238} \mathrm{U}$ sugiere que otros carbonatos biogénicos, por ejemplo conchas o caparazones, son candidatos altamente atractivos para ser fechados por series de U. Sin embargo, la cercanía de los sujetos generadores del carbonato a ambientes ricos en material detrítico (suelos y sedimentos) dificulta la datación precisa debido a la alta proporción de ${ }^{232} \mathrm{Th}$ de origen detrítico que se incorpora en dichos carbonatos.

Otras fases minerales que han sido fechados por series de $\mathrm{U}$ incluyen ópalo y sílice pedogenética (Neymark y Paces, 2000; Ludwig y Paces, 2002) y óxi-hidróxidos de Fe formados durante procesos de intemperismo (Bernal et al., 2006). En ambos casos la datación de dichas fases permitió establecer el papel que juegan las condiciones climáticas globales sobre las tasas de intemperismo y pedogénesis. Por otra parte, se han realizado esfuerzos por fechar yeso evaporítico procedente de núcleos de sedimentos lacustres (Gamble et al., 2007). Sin embargo, la alta concentración de $\mathrm{Th}$ en los sedimentos hace que las correcciones por contribución de material detrítico no sean factibles. Asimismo, minerales secundarios de $\mathrm{U}$ de la familia de la carnotita, $\mathrm{K}_{2}\left(\mathrm{UO}_{2}\right)_{2}\left(\mathrm{VO}_{4}\right)_{2} \cdot 3 \mathrm{H}_{2} \mathrm{O}$, formados a partir del intemperismo químico de la uraninita han sido datados también por series de U (Kaufman y Ku, 1989; Kaufman et al., 1995).

La datación de materiales de origen volcánico por series de $U$ fue una de las primeras aplicaciones donde el desequilibrio en la cadena de decaimiento del ${ }^{238} \mathrm{U}$ fue utilizada con fines geocronológicos (Allegre, 1968; Allegre y Condomines, 1976). Lo anterior debido a que, a diferencia del fechado de fases minerales secundarias, la cantidad de material contemporáneo disponible para el análisis por espectrometría $\alpha$ es mucho mayor. De esta manera es posible obtener resultados mucho más confiables y geocronológicamente más significativos a partir del análisis de estos materiales por espectrometría $\alpha$. Aunque las series de $\mathrm{U}$ han sido aprovechadas para fechar eventos volcánicos del Cuaternario desde hace varios años, una de sus principales aplicaciones en la actualidad es para establecer limitaciones geocronológicas a diversos procesos de diferenciación al interior de cámaras magmáticas (Condomines et al., 2003), así como el establecimiento de tiempos de residencia del magma (Sigmarsson et al., 2005). A diferencia de los procesos de diferenciación a baja temperatura, no se ha observado fraccionamiento entre ${ }^{234} \mathrm{U} /{ }^{238} \mathrm{U}$ en fases minerales volcánicas jóvenes. Lo anterior implica que el cálculo de edad a partir de la Ecuación 15 puede simplificarse ya que $\left[{ }^{234} U /{ }^{238} U\right]$ será $=1$, o $\delta 234=$ 0, quedando: 


$$
\begin{aligned}
& {\left[\frac{{ }^{230} \mathrm{Th}}{{ }^{238} \mathrm{U}}\right]=1-\mathrm{e}^{-\lambda_{230} \mathrm{t}}} \\
& \mathrm{o} \\
& {\left[\frac{{ }^{230} \mathrm{Th}}{{ }^{238} \mathrm{U}}\right]=1-\mathrm{e}^{-\lambda_{230} \mathrm{t}}}
\end{aligned}
$$

Ecuación 16

Los isótopos de $\mathrm{U}$ y Th son también utilizados para la datación de fósiles óseos recientes (Pike y Hedges, 2001; Belshaw et al., 2002; Pike et al., 2002), así como indicadores de procesos de sedimentación y condiciones paleo-oceanográficas (Henderson, 2002), intempersimo (Dequincey et al., 2002; Chabaux et al., 2003), interacción agua-partícula en acuíferos (Porcelli et al., 1997, 2001; Tricca et al., 2001).

\subsection{Metodologías analíticas}

Desde el punto de vista analítico, la cuantificación de ${ }^{234} \mathrm{U} \mathrm{y}^{230} \mathrm{Th}$ es uno de los retos más difíciles para cualquier laboratorio de geocronología debido a la baja abundancia y actividad de estos isótopos. La concentración promedio del $\mathrm{U}$ en la corteza terrestre es de tan sólo $2.7 \mathrm{mg} / \mathrm{kg} \mathrm{y}$, en las condiciones más favorables, la relación isotópica ${ }^{230} \mathrm{Th} /{ }^{238} \mathrm{U}$ $=1.695 \times 10^{-5}$. Lo anterior implica que en una roca promedio la concentración de ${ }^{230} \mathrm{Th}$ puede llegar a ser, cuando mucho, unos cuantos $\mathrm{ng} / \mathrm{kg}$, mientras que el ${ }^{234} \mathrm{U}$ puede estar unas 4 o 5 veces más concentrado que el ${ }^{230} \mathrm{Th}$. De esta manera, la cuantificación de estos isótopos requiere infraestructura analítica de muy alta sensibilidad y condiciones de trabajo de gran limpieza.

Independientemente de la técnica instrumental utilizada para cuantificar U y Th, la separación y preconcentración de U y Th de la matriz en la que se encuentran es esencial para la remoción de interferencias en el rango espectral o de masas de los isótopos de U. Lo anterior se lleva a cabo después de la disolución de la muestra de diversas maneras, que incluye co-precipitación con $\mathrm{Fe}(\mathrm{OH})_{3}$ y re-disolución en $\mathrm{HCl}$ para después purificar el U y Th utilizando técnicas de intercambio iónico con resinas poliméricas, tales como TRU-Spec $\AA$ y UTEVA $®($ Kim et al., 2000). Como resultado del proceso de purificación se obtiene, por lo general, un concentrado de $\mathrm{U}$ y otro de Th, los cuales se miden generalmente de manera independiente utilizando dilución isotópica.

Durante más de 40 años la cuantificación de ${ }^{234} \mathrm{U}$ y ${ }^{230}$ Th se llevó a cabo a partir del análisis del espectro de emisión de radiación $\alpha$ emitida por estos isótopos o sus isótopos hijos de mayor actividad (Ivanovich y Murray, 1992). Dicha aproximación demostró ser de gran utilidad para el análisis y datación de especimenes donde no había limitación en la cantidad de muestra disponible, por ejemplo, rocas volcánicas jóvenes o sus concentrados minerales.
Sin embargo, debido a que la espectrometría $\alpha$ requiere cuando menos $100 \mu \mathrm{g}$ de $\mathrm{U}$, esta técnica muestra severas limitaciones para el fechado de especimenes pequeños, por ejemplo corales o estalagmitas, donde las tasas bajas de acumulación no permiten colectar suficiente material contemporáneo. De esta manera, incertidumbres en la edad cercanas al $\pm 10 \%(1 \sigma)$ son típicas para las dataciones U-Th de carbonatos secundarios utilizando espectrometría $\alpha$ (p. ej: Latham et al., 1986).

La datación de fases minerales secundarias por U-Th únicamente fue factible hasta que se establecieron las metodologías analíticas para la determinación de ${ }^{234} \mathrm{U}$ y ${ }^{230} \mathrm{Th}$ por espectrometría de masas con ionización térmica -TIMS (Edwards et al., 1987). A partir de entonces, se han realizado diversas mejoras instrumentales en espectrometría de masas para incrementar la eficiencia de ionización, transporte y detección de iones. La introducción de plasmas inductivamente acoplados (ICP) como fuente de ionización en espectrometría de masas (Houk et al., 1980) y su incorporación a equipos de sector magnético multicolectores MC-ICPMS (Bradshaw et al., 1989) es, posiblemente, uno de los avances instrumentales más significativos en geocronología. Debido a que el ICP se encuentra a $\sim 10000$ $\mathrm{K}$ es una fuente de iones monocargados de alta eficiencia, lo cual simplificó de manera significativa el análisis de U, Th y sus isótopos (Luo et al., 1997). De esta manera, y debido a la gran sensibilidad del MC-ICPMS, los desarrollos instrumentales han estado enfocados hacia la datación de muestras que se encuentran cercanas al equilibrio secular (Andersen et al., 2004), así como muestras extremadamente jóvenes (McCulloch y Mortimer, 2008). Por otra parte, el acoplamiento de sistemas de ablación láser a MC-ICPMS (Stirling et al., 2000; Bernal et al., 2005) ha permitido realizar dataciones bajo un contexto espacial y en texturas minerales que no permiten la separación de física o química de los minerales (Bernal et al., 2006), o bien, simplificar significativamente los procesos de preparación de muestra para el análisis de carbonatos para poder realizar análisis geocronológicos exploratorios previos a la datación detallada (Potter et al., 2005b).

Con excepción de las metodologías de ablación láser, la cuantificación de los isótopos de U y Th se lleva a cabo siguiendo metodologías de dilución isotópica. Lo anterior implica la adición de un trazador de manera previa a los ensayos de separación cromatográfica del U y el Th. Por lo general, el análisis de isótopos de U por espectrometría $\alpha$ requiere la adición de ${ }^{232} \mathrm{U} \mathrm{y}^{228} \mathrm{Th}$, cuyas vidas medias cortas (70 y 1.9 años, respectivamente) permite cuantificarlos a partir de su espectro de decaimiento $\alpha$ con gran precisión. Para la medición por espectrometría de masas se utilizan isótopos de mayor estabilidad, tales como ${ }^{233} \mathrm{U}-\left({ }^{236} \mathrm{U}\right)$ y ${ }^{229} \mathrm{Th}$, con vidas medias de $1.6 \times 10^{5}, 2.3 \times 10^{7}$ y $7.9 \times 10^{3}$ años, respectivamente). Lo anterior requiere que se lleve a cabo un proceso de calibración del trazador, tanto de manera gravimétrica como por su composición isotópica, la cual se puede llevar a cabo por dilución isotópica inversa de 
estándares gravimétricos de U y Th o por el análisis de materiales con composición isotópica conocida

\section{Conclusiones}

En este documento se han descrito brevemente tres de los métodos de datación radiométrica más utilizados para generar cronologías del Cuaternario, con el objetivo de que el lector tenga un panorama general de los fundamentos de cada uno y pueda, en su caso, seleccionar las muestras apropiadas e interpretar los resultados para generar una cronología lo más precisa posible. Sin embargo, hay otras metodologías radiométricas que cada vez se utilizan con mayor frecuencia para el estudio de procesos geológicos y ambientales ocurridos en los últimos $2.8 \mathrm{Ma}$ entre las que se incluyen los sistemas de U/Pb, K/Ar y ${ }^{40} \mathrm{Ar} /{ }^{39} \mathrm{Ar}$. Por otra parte, otros métodos como la termoluminiscencia (la luminiscencia estimulada en granos de cuarzo) también fundamentados en el decaimiento radioactivo de U, Th y K del medio alrededor de la muestra, cobran cada vez mayor importancia como métodos de datación de sedimentos, suelos y material arqueológico. El lector deberá referirse a la literatura más especializada (p. ej: Noller et al., 2000) para profundizar en los principios establecidos en este documento.

\section{Referencias}

Alcaraz Pelegrina, J.M., Martínez-Aguirre, A., 2005, Isotopic fractionation during leaching of impure carbonates and their effect on uranium series dating: Quaternary Science Reviews, 24, 2584-2593.

Allegre, C.J., 1968, ${ }^{230} \mathrm{Th}$ dating of volcanic rocks: a comment: Earth and Planetary Science Letters, 5, 209-210.

Allegre, C.J., Condomines, M., 1976, Fine chronology of volcanic processes using 238U-230Th systematics: Earth and Planetary Science Letters, 28, 395-406.

Andersen, M.B., Stirling, C.H., Potter, E.-K., Halliday, A.N., 2004, Toward epsilon levels of measurement precision on ${ }^{234} \mathrm{U} /{ }^{238} \mathrm{U}$ by using MCICPMS: International Journal of Mass Spectrometry, 237, 107-118.

Appleby, P.G., 2001, Chronostratigraphic techniques in recent sediments, en Last, W.M., Smol, J.P. (eds.), Tracking Environmental Change Using Lake Sediments Volume 1 Basin Analysis, Coring, and Chronological Techniques: Dordrecht, Países Bajos, Kluwer Academic, 171-203.

Appleby, P.G., Oldfield, F., 1978, The calculation of ${ }^{210} \mathrm{~Pb}$ dates assuming a constant rate of supply of unsupported ${ }^{210} \mathrm{~Pb}$ to the sediment: Catena, 5, 1-8.

Appleby, P.G., Oldfield, F., 1983, The assessment of ${ }^{210} \mathrm{~Pb}$ data from sites with varying sediment accumulation rates: Hydrobiologia, 103, 29-35.

Audi, G., Bersillon, O., Blachot, J., Wapstra, A.H., 1997, The NUBASE evaluation of nuclear and decay properties: Nuclear Physics A, $624,1-124$

Ayling, B.F., McCulloch, M.T., Gagan, M.K., Stirling, C.H., Andersen, M.B., Blake, S.G., 2006, $\mathrm{Sr} / \mathrm{Ca}$ and delta ${ }^{18} \mathrm{O}$ seasonality in a Porites coral from the MIS 9 (339-303 ka) interglacial: Earth and Planetary Science Letters, 248, 462-475.

Baker, A., Smart, P.L., Edwards, R.L., Richards, D.A., 1993, Annual Growth Banding in a Cave Stalagmite: Nature, 364, 518-520.

Bard, E., 1998, Geochemical and geophysical implications of the radiocarbon calibration: Geochimica et Cosmochimica Acta, 62, 2025-2038.

Belshaw, N.S., Pike, A.W.G., Henderson, G.M., 2002, U-series dating of archaeological bone material by laser ablation multiple ion counter ICP-MS (abstract): Geochimica et cosmochimica acta, 66, A65.

Benoit, G., Hemod, H.F., 1991, Evidence for diffusive redistribution redistribution of ${ }^{210} \mathrm{~Pb}$ in lake sediments: Geochimica et Cosmochimica Acta, 55, 1963-1975.

Bernal, J.P., Railsback, L.B., 2008, Introducción a la Tabla Periódica de los Elementos y sus Iones para Ciencias de la Tierra: Revista Mexicana de Ciencias Geológicas, 25, 236-246.

Bernal, J.P., Eggins, S.M., McCulloch, M.T., 2005, Accurate in situ ${ }^{238} \mathrm{U}^{234} \mathrm{U}^{232} \mathrm{Th}-{ }^{230} \mathrm{Th}$ analysis of silicate glasses and iron oxide by laser-ablation MC-ICP-MS: Journal of Analytical Atomic Spectrometry, 20, 1240-1249.

Bernal, J.P., Eggins, S.M., McCulloch, M.T., Grün, R., Eggleton, R.A., 2006, Dating of chemical weathering processes by in situ measurement of U-series disequilibria in supergene Fe-oxy/ hydroxides using LA-MC-ICPMS: Chemical Geology, 235, 76-94.

Bischoff, J.L., Fitzpatrick, J.A., 1991, U-series dating of impure carbonates: An isochron technique using total-sample dissolution: Geochimica et Cosmochimica Acta, 55, 543-554.

Bourdon, B., Turner, S., Henderson, G.M., Lundstrom, C.C., 2003, Introduction to U-series geochemistry, en Bourdon, B., Turner, S.P., Henderson, G.M., Lundstrom, C.C., Turner, S.P. (eds.), Uraniumseries geochemistry: Washington D.C., Geochemical SocietyMineralogical Society of America, 1-20.

Bradshaw, N., Hall, E.F.H., Sanderson, N.E., 1989, Inductively coupled plasma as an ion source for high-resolution mass spectrometry: Journal of Analytical Atomic Spectrometry, 4: 801.

Broecker, W.S., 1963, A preliminary evaluation of uranium series inequilibrium as a tool for absolute age measurement on marine carbonates: Journal of Geophysical Research, 68, 2817-2834.

Bronk Ramsey, C., 2008, Radiocarbon dating: Revolutions in understanding: Archaeometry, 50, 249-275.

Carroll, J., Lerche, I., 2003, Sedimentary processes quantification using radionuclides: Amsterdam, Elsevier, $272 \mathrm{p}$.

Cobb, K.M., Charles, C.D., Cheng, H., Kastner, M., Lawrence Edwards, R., 2003, U/Th-dating living and young fossil corals from the central tropical Pacific: Earth and Planetary Science Letters, 210, 91-103.

Condomines, M., Gauthier, P.-J. Sigmarsson, O., 2003, Timescales of magma chamber processes and dating of young volcanic rocks, en Bourdon, B., Henderson, G.M., Lundstrom, C.C., Turner, S.P. (eds.), Uranium-Series Geochemistry: Washington, D.C., Geochemical Society-Mineralogical Society of America, 125-174.

Craig, H., 1954, Carbon 13 in plants and the relationships between carbon 13 and carbon 14 variations in nature: Journal of Geology, 62, 115-149.

Chabaux, F., Dequincey, O., Leveque, J.J., Leprun, J.C., Clauer, N., Riotte, J., Paquet, H., 2003, Tracing and dating recent chemical transfers in weathering profiles by trace-element geochemistry and ${ }^{238} \mathrm{U}_{-}{ }^{234} \mathrm{U}-$ ${ }^{230} \mathrm{Th}$ disequilibria: the example of the Kaya lateritic toposequence (Burkina-Faso): Comptes Rendus Geosciences, 335, 1219-1231.

Cheng, H., Adkins, J., Lawrence Edwards, R., Boyle, E.A., 2000a, U-Th dating of deep-sea corals: Geochimica et Cosmochimica Acta, 64, 2401-2416.

Cheng, H., Edwards, R.L., Hoff, J., Gallup, C.D., Richards, D.A., Asmerom, Y., 2000b, The half-lives of uranium-234 and thorium-230: Chemical Geology, 169, 17-33.

Cherdynstsev, V.V., 1955, Uranium series disequilibrium dating, en Transactions of the 3rd Session of the Commission for determining the absolute age of geological formations: Izd. Akad. Nauk., Moscú, URSS, 175-182.

Dequincey, O., Chabaux, F., Clauer, N., Sigmarsson, O., Liewig, N., Leprun, J.-C., 2002, Chemical mobilizations in laterites: evidence from trace elements and ${ }^{238} \mathrm{U}_{-}{ }^{234} \mathrm{U}_{-}{ }^{230} \mathrm{Th}$ disequilibria: Geochimica et Cosmochimica Acta, 66, 1197-1210.

Dickin, A.P., 1995, Radiogenic isotope geology: Cambridge, Inglaterra, Cambridge University Press, 495 p. 
Dorale, J.A., Edwards, R.L., Calvin Alexander, J., Shen, C.-C., Richards, D.A., Cheng, H., 2004, Uranium-series dating of speleothems: Current techniques, limits, and applications, en Sasowsky, I.D., Mylroie, J. (eds.), Studies of cave sediments, physical and chemical records of paleoclimate: New York, Kluwer Academic, 177-198.

Edwards, R.L., Chen, J.H., Wasserburg, G.J., 1987, ${ }^{238} \mathrm{U}^{234} \mathrm{U}^{230} \mathrm{Th}-{ }^{232} \mathrm{Th}$ systematics and the precise measurement of time over the past 500,000 years: Earth and Planetary Science Letters, 81, 175-192.

Eikenberg, J., Vezzu, G., Zumsteg, I., Bajo, S., Ruethi, M., Wyssling, G., 2001, Precise two chronometer dating of Pleistocene travertine: The ${ }^{230} \mathrm{Th} /{ }^{234} \mathrm{U}$ and ${ }^{226} \mathrm{Ra}_{\text {ex }}{ }^{226} \mathrm{Ra}_{(0)}$ approach: Quaternary Science Reviews, 20, 1935-1953.

Esat, T.M., McCulloch, M.T., Chappell, J., Pillans, B., Omura, A., 1999, Rapid fluctuations in sea level recorded at Huon Peninsula during the penultimate deglaciation: Science, 283, 197-201.

Fleischer, R.L., 1980, Isotopic disequilibrium of uranium: alpha-recoil damage and preferential solution effects: Science, 207, 979-981.

Fleischer, R.L., 1982, Alpha-recoil damage and solution effects in minerals uranium isotopic disequilibrium and radon release: Geochimica et Cosmochimica Acta, 46, 2191-2201.

Fletcher, K.E.K., Sharp, W.D., Kendrick, K.J., Behr, W.M., Hudnut, K.W., Hanks, T.C., 2010, ${ }^{230} \mathrm{Th} / \mathrm{U}$ dating of a late Pleistocene alluvial fan offset along the southern San Andreas fault: Geological Society of America Bulletin, 122, 1347-1359.

Gallup, C.D., Cheng, H., Taylor, F.W., Edwards, R.L., 2002, Direct Determination of the Timing of Sea Level Change During Termination II: Science, 295, 310-313.

Gamble, V.C., Gallup, C.D., Hodell, D.A., Anselmentti, F., Brenner, M., 2007, Preliminary U-Th dating of endogenic gypsum from Lake Petén-Itzá, Guatemala (abstract): Eos Transactions AGU Joint Assembly Supplement, 88, PP43C-03.

Godwin, H., 1962, Half-Life of Radiocarbon: Nature, 195, 984-987.

Goldberg, E.D., Koide, M., 1962, Geochronological studies of deep sea sediments by the ionium/thorium method: Geochimica et Cosmochimica Acta, 26, 417-450.

Hajdas, I., 2009, Applications of Radiocarbon Dating Method: Radiocarbon, 51: 79-90.

Hedges, R.E.M., 1992, Sample treatment strategies in radiocarbon dating, en Taylor, R.E., Long, A., Kra, R.S. (eds.), Radiocarbon after four decades. An interdisciplinary perspective: Nueva York, SpringerVerlag, 165-183.

Henderson, G.M., 2002, Seawater $\left({ }^{234} \mathrm{U}{ }^{238} \mathrm{U}\right)$ during the last 800 thousand years: Earth and Planetary Science Letters, 199, 97-110.

Hogg, A.G., Fifield, L.K., Turney, C.S.M., Palmer, J.G., Galbraith, R., Baillie, M.G.K., 2006, Dating ancient wood by high-sensitivity liquid scintillation counting and accelerator mass spectrometry Pushing the boundaries: Quaternary Geochronology, 1, 241-248.

Horvatincic, N., Calic, R., Geyh, M.A., 2000, Interglacial growth of tufa in Croatia: Quaternary Research, 53, 185-195.

Houk, R.S., Fassel, V.A., Flesch, G.D., Svec, H.J., Gray, A.L., Taylor, C.E., 1980, Inductively coupled argon plasma as an ion source for mass spectrometric determination of trace elements: Analytical Chemistry, 52, 2283-2289.

Ivanovich, M., Harmon, R.S., 1992, Uranium-series disequilibrium applications to earth, marine, and environmental science: Oxford, Inglaterra, Oxford-Clarendon Press, $910 \mathrm{p}$.

Ivanovich, M., Murray, A., 1992, Spectroscopic methods, en Ivanovich, M., Harmon, R.S., (eds.), U-series disequilibrium Applications to earth, marine, and environmental sciences: Oxford, Inglaterra, Oxford-Clarendon Press, 127-173.

Jaffey, A.H., Flynn, K.F., Glendenin, L.E., Bentley, W.C., Essling, A.M., 1971, Precision measurement of half-lives and specific activities of ${ }^{235} \mathrm{U}$ and ${ }^{238} \mathrm{U}$ : Physical Review C, 4, 1889-1906.

Kaufman, A., 1993, An evaluation of several methods for determining $230 \mathrm{Th} / \mathrm{U}$ ages in impure carbonates: Geochimica et Cosmochimica Acta, 57, 2303-2317.

Kaufman, A., Ku, T.L., 1989, The U-series ages of carnotites and implications regarding their formation: Geochimica et Cosmochimica
Acta, 53, 2675-2681.

Kaufman, A., Ku, T.L., Luo, S., 1995, Uranium-series dating of carnotites: concordance between ${ }^{230} \mathrm{Th}$ and ${ }^{231} \mathrm{~Pa}$ ages: Chemical Geology, 120 , $175-181$.

Kigoshi, K., 1971, Alpha-recoil Thorium-234: dissolution into water and the Uranium-234/Uranium-238 disequilibrium in nature: Science, $173,47-48$.

Kim, G., Burnett, W.C., Horwitz, E.P., 2000, Efficient preconcentration and separation of actinide elements from large soil and sediment samples: Analytical Chemistry, 72, 4882-4887.

Koide, M., Soutar, A., Goldberg, E.D., 1972, Marine Geochronology with ${ }^{210} \mathrm{~Pb}$ : Earth and Planetary Science Letters, 14, 442-446.

Krishnaswamy, S., Lal, D., 1978, Radionuclide Limnochronology, en Lerman, A. (ed.), Lakes Chemistry, Geology and Physics: New York, Springer Verlag, 153-177.

Krishnaswamy, S., Lal, D., Martin, J.M., Meybek, M., 1971. Geochronology of lake sediments: Earth and Planetary Science Letters, 11: 407-414.

Lambeck, K., Esat, T.M., Potter, E.K., 2002, Links between climate and sea levels for the past three million years: Nature, 419, 199-206.

Langmuir, D., 1978, Uranium solution-mineral equilibria at low temperatures with applications to sedimentary ore deposits: Geochimica et Cosmochimica Acta, 42, 547-569.

Langmuir, D., Herman, J.S., 1980, The mobility of thorium in natural waters at low temperatures: Geochimica et Cosmochimica Acta, 44, 1753-1766.

Latham, A.G., Schwarcz, H.P., Ford, D.C., 1986, The paleomagnetism and U-Th dating of Mexican stalagmite, DAS2: Earth and Planetary Science Letters, 79, 195-207.

Libby, W.F., 1955, Radiocarbon Dating: Chicago, Illinois, EUA, University of Chicago Press: $175 \mathrm{p}$.

Lowe, J.J., Walker, M.J.C., 2000, Radiocarbon dating the last glacialinterglacial transition (Ca. $\left.14-9{ }^{14} \mathrm{C} \mathrm{ka} \mathrm{BP}\right)$ in terrestrial and marine records: The need for new quality assurance protocols: Radiocarbon, 42, 53-68.

Ludwig, K.R., 2003, Mathematical-statistical treatment of data and errors for $230 \mathrm{Th} / \mathrm{U}$ geochronology, en Bourdon, B., Henderson, G.M., Lundstrom, C.C., Turner, S. P. (eds.), Uranium-Series Geochemistry: Washington, D.C., Geochemical Society-Mineralogical Society of America, 631-656.

Ludwig, K.R., Paces, J.B., 2002, Uranium-series dating of pedogenic silica and carbonate, Crater Flat, Nevada: Geochimica et Cosmochimica Acta, 66, 487-506.

Ludwig, K.R., Titterington, D.M., 1994, Calculation of ${ }^{230} \mathrm{Th} / \mathrm{U}$ isochrons, ages and errors: Geochimica et Cosmochimica Acta, 58, 5031-5042.

Ludwig, K.R., Simmons, K.R., Szabo, B.J., Winograd, I.J., Landwehr, J.M., Riggs, A.C., Hoffman, R.J., 1992, Mass-spectrometric ${ }^{230}{ }^{20}{ }^{234}{ }^{23}-$ ${ }^{238} \mathrm{U}$ dating of the Devils Hole Calcite Vein: Science, 258, 284-287.

Ludwig, K.R., Szabo, B.J., Moore, J.G., Simmons, K.R., 1991, Crustal subsidence rate off Hawaii determined from ${ }^{234} \mathrm{U} /{ }^{238} \mathrm{U}$ ages of drowned coral reefs: Geology, 19, 171-174.

Luo, S., Ku, T.L., 1991, U-series isochron dating: A generalized method employing total-sample dissolution: Geochimica et Cosmochimica Acta, 55, 555-564.

Luo, X., Rehkamper, M., Lee, D.C., Halliday, A.N., 1997, High precision ${ }^{230} \mathrm{Th} /{ }^{232} \mathrm{Th}$ and ${ }^{234} \mathrm{U} /{ }^{238} \mathrm{U}$ measurements using energy filtered ICP magnetic sector multiple collector mass spectrometry: International Journal of Mass Spectrometry and Ion Processes, 171, 105-117.

Marques, A.N., Monna, F., da Silva Filho, E.V., Fernex, F.E., Lamego Simões, F.F., 2006, Apparent discrepancy in contamination history of a sub-tropical estuary evaluated through ${ }^{210} \mathrm{~Pb}$ profile and chronostratigrafical markers: Marine Pollution Bulletin, 52, 532-539.

McCulloch, M.T., Mortimer, G.E., 2008, Applications of the ${ }^{238} \mathrm{U}_{-}^{230} \mathrm{Th}$ decay series to dating of fossil and modern corals using MC-ICPMS: Australian Journal of Earth Sciences, 55, 955-965.

McCulloch, M.T., Tudhope, A.W., Esat, T.M., Mortimer, G.E., Chappell, J., Pillans, B., Chivas, A.R., Omura, A., 1999, Coral record of equatorial sea-surface temperatures during the penultimate deglaciation at Huon Peninsula: Science, 283, 202-204. 
McCulloch, M., Fallon, S., Wyndham, T., Hendy, E., Lough, J., Barnes, D., 2003, Coral record of increased sediment flux to the inner Great Barrier Reef since European settlement: Nature, 421, 727-730.

McFadgen, B.G., Knox, F.B., Cole, T.R.L., 1994, Radiocarbon calibration curve variations and their Implications for the Interpretation of New Zealand Prehistory: Radiocarbon, 36, 221-236.

Neymark, L.A., Paces, J.B., 2000, Consequences of slow growth for ${ }^{230} \mathrm{Th} / \mathrm{U}$ dating of Quaternary opals, Yucca Mountain, NV, USA: Chemical Geology, 164, 143-160.

Noller, J.S., Sowers, J.M., Lettis, W.R., 2000, Quaternary geochronology: methods and applications: Washington D.C., American Geophysical Union, $582 \mathrm{p}$.

Oldfield, F., Appleby, P.G., 1985, Empirical testing of ${ }^{210} \mathrm{~Pb}$-dating models for lake sediments, en Haworth, E.Y., Lund, J.W.G. (eds.), Lake Sediments and Environmental History: Minneapolis, Minnesota, EUA, University of Minnesota Press, 93-124.

Pennington, W., Tutin, T.G., Cambray, R.S., Fisher, E.M., 1973, Observations on lake sediments using fallout ${ }^{137} \mathrm{Cs}$ as a tracer: Nature, 242, 324-326.

Pike, A.W.G., Hedges, R.E.M., 2001, Sample geometry and U uptake in archaeological teeth: implications for U-series and ESR dating: Quaternary Science Reviews, 20, 1021-1025.

Pike, A.W.G., Hedges, R.E.M., Van calsteren, P., 2002, U-series dating of bone using the diffusion-adsorption model: Geochimica et Cosmochimica Acta, 66, 4273-4286.

Porcelli, D., Andersson, P.S., Wasserburg, G.J., Ingri, J., Baskaran, M., 1997, The importance of colloids and mires for the transport of uranium isotopes through the Kalix River watershed and Baltic Sea: Geochimica et Cosmochimica Acta, 61, 4095-4113.

Porcelli, D., Andersson, P.S., Baskaran, M. Wasserburg, G.J., 2001, Transport of U- and Th-series nuclides in a Baltic shield watershed and the Baltic sea: Geochimica et Cosmochimica Acta, 65, 24392459.

Potter, E.K., Stirling, C.H., Andersen, M.B., Halliday, A.N., 2005a, High precision Faraday collector MC-ICPMS thorium isotope ratio determination: International Journal of Mass Spectrometry, 247, 10-17.

Potter, E.K., Stirling, C.H., Wiechert, U.H., Halliday, A.N., Spotl, C., 2005b, Uranium-series dating of corals in situ using laser-ablation MC-ICPMS: International Journal of Mass Spectrometry, 240, 27-35.

Pourchet, M., Pinglot, J.F., Gascard, J.C., 1986, The northly extent of Chernobyl contamination: Nature, 323, 676-677.

Pourchet, M., Pinglot, J.F., Reynaud, L., Holdsworth, G., 1988, Identification of Chernobyl fall-out as a new reference level in Northern Hemisphere glaciers: Journal of Glaciology, 34, 183-187.

Reimer, P.J., Baillie, M.G.L., Bard, E., Bayliss, A., Beck, J.W., Bertrand, C.J.H., Blackwell, P.G., Buck, C.E., Burr, G.S., Cutler, K.B., Damon, P.E., Edwards, R.L., Fairbanks, R.G., Friedrich, M., Guilderson, T.P., Hogg, A.G., Hughen, K.A., Kromer, B., McCormac, G., Manning, S., Bronk Ramsey, C., Reimer, R.W., Remmele, S., Southon, J.R., Stuiver, M., Talamo, S., Taylor, F.W., Plicht, J., Weyhenmeyer, C.E., 2004, IntCal04 terrestrial radiocarbon age calibration, 0-26 Cal kyr BP: Radiocarbon, 46: 1029-1058.

Robbins, J.A., Edgington, D.N., 1975, Determination of recent sedimentation rates in Lake Michigan using Pb-210 and Cs-137: Geochimica et Cosmochimica Acta, 39, 285-304.

Robbins, R.A., 1978, Geochemical and geophysical application of radioactive lead, en Nriagu, J.O. (ed.), The biogeochemistry of lead in the environment: Amsterdam, Elsevier Publishing, 286-383.

Rosholt, J.N., 1976, ${ }^{230} \mathrm{Th} /{ }^{234} \mathrm{U}$ dating of travertine and caliche rinds: Geological Society of America - Abstracts with Programs, 8, 1076

Rosholt, J.N., Tatsumoto, M., 1970, Isotopic composition of uranium and thorium in Apollo 11 samples. Proceedings of the Apollo 11 Lunar Science conference: Geochimica et Cosmochimica Acta Supplement, $1,1499-1502$.

Rosholt, J.N., Tatsumoto, M., 1971, Isotopic composition of thorium and uranium in Apollo 12 samples. Proceedings Apollo 12 Lunar Science conference: Geochimica et Cosmochimica Acta Supplement,
2, 1577-1584.

Santschi, P.H., Li, Y.H., Bell, J., Adler, D.M., Amdurer, M., Bell, J., Nyffeler, U.P., 1983, The relative mobility of natural (Th, Pb and $\mathrm{Po}$ ) and fallout $(\mathrm{Pu}, \mathrm{Am}, \mathrm{Cs})$ radionuclides in the coastal marine environment: results from model ecosystems (MERL) and Narragansett Bay: Geochimica et Cosmochimica Acta, 47, 201-210.

Schwarcz, H.P., Latham, A.G., 1989, Dirty calcites 1. Uranium-series dating of contaminated calcite using leachates alone: Chemical Geology, 80, 35-43.

Sharp, W.D., Ludwig, K.R., Chadwick, O.A., Amundson, R., Glaser, L.L., 2003, Dating fluvial terraces by ${ }^{230} \mathrm{Th} / \mathrm{U}$ on pedogenic carbonate, Wind River Basin, Wyoming: Quaternary Research, 59, 139-150.

Sigmarsson, O., Condomines, M., Bachelery, P., 2005, Magma residence time beneath the Piton de la Fournaise Volcano, Reunion Island, from U-series disequilibria: Earth and Planetary Science Letters, 234, 223-234.

Smith, J.N., 2001, Why should we believe ${ }^{210} \mathrm{~Pb}$ sediment geochronologies?: Journal of Environmental Radioactivity, 55, 121-123.

Srdoc, D., Osmond, J.K., Horvatincic, N., Dabous, A.A., Obelic, B., 1994, Radiocarbon and Uranium-Series dating of the Plitvice Lakes travertines: Radiocarbon, 36, 203-219.

Staunton, S., 2002, Direct and indirect effects of organic matter on metal immobilisation in soil, en Violante, A. (ed.), Soil Mineral-Organic Matter-Microorganism interactions and ecosystem health Volume 28B. Ecological significance of the interactions among clay minerals, organic matter and soil biota: Amsterdam, Elsevier, 79-97.

Stirling, C.H., Esat, T.M., McCulloch, M.T., Lambeck, K., 1995, Highprecision U-series dating of corals from Western Australia and implications for the timing and duration of the last interglacial: Earth and Planetary Science Letters, 135, 115-130.

Stirling, C.H., Lee, D.C., Christensen, J.N., Halliday, A.N., 2000, Highprecision in $\operatorname{situ}^{238} \mathrm{U}-{ }^{234} \mathrm{U}-{ }^{230} \mathrm{Th}$ isotopic analysis using laser ablation multiple-collector ICPMS: Geochimica et Cosmochimica Acta, 64, 3737-3750.

Stirling, C.H., Esat, T.M., Lambeck, K., McCulloch, M.T., Blake, S.G., Lee, D.C., Halliday, A.N., 2001, Orbital forcing of the marine isotope stage 9 interglacial: Science, 291, 290-293.

Stuiver, M., Polach, H.A., 1977, Discussion: Reporting of ${ }^{14} \mathrm{C}$ data: Radiocarbon, 19, 355-363.

Szabo, B.J., O'Malley, P.A., 1985, Uranium-series dating of secondary carbonate and silica precipitates relating to fault movements in the Nevada Test Site region and of caliche and travertine samples from the Amargosa Desert: Denver, Colorado, U.S. Geological Survey, $12 \mathrm{p}$.

Takahashi, Y., Sakurai, H., Suzuki, K., Sato, T., Gunji, S., Tokanai, F., Matsuzaki, H., Sunohara, Y., 2009, Comparison of ${ }^{14} \mathrm{C}$ ages between LSC and AMS measurements for Choukai Jindai cedar tree rings at the rapid change in 2600 cal BP (resumen), en 20th International Radiocarbon Conference: Big Island, Hawaii, EUA, 36.

Taylor, R.E., 1987, Radiocarbon dating: An archaeological perspective: Londres, Academic Press, 212 p.

Tricca, A., Wasserburg, G.J., Porcelli, D., Baskaran, M., 2001, The transport of $\mathrm{U}$ - and Th-series nuclides in a sandy unconfined aquifer: Geochimica et Cosmochimica Acta, 65, 1187-1210.

Wang, Y.J., Cheng, H., Edwards, R.L., An, Z.S., Wu, J.Y., Shen, C.-C. Dorale, J.A., 2001, A high-resolution absolute-dated Late Pleistocene monsoon record from Hulu Cave, China: Science, 294, 2345-2348.

Wang, Y., Cheng, H., Edwards, R.L., He, Y., Kong, X., An, Z., Wu, J., Kelly, M.J., Dykoski, C.A., Li, X., 2005, The Holocene Asian monsoon: links to solar changes and North Atlantic climate: Science, 308, 854-857.

Wang, X., Auler, A.S., Lawrence Edwards, R., Cheng, H., Ito, E., Solheid, M., 2006, Interhemispheric anti-phasing of rainfall during the last glacial period: Quaternary Science Reviews, 25, 3391-3403.

Winograd, I.J., Coplen, T.B., Landwehr, J.M., Riggs, A.C., Ludwig, K.R., Szabo, B.J., Kolesar, P.T., Revesz, K.M., 1992, Continuous 500,000year climate record from vein calcite in Devils-Hole, Nevada: Science, 258, 255-260. 
Winograd, I.J., Landwehr, J.M., Ludwig, K.R., Coplen, T.B., Riggs, A.C., 1997, Duration and Structure of the Past Four Interglaciations: Quaternary Research, 48, 141-154.

Winograd, I.J., Landwehr, J.M., Coplen, T.B., Sharp, W.D., Riggs, A.C., Ludwig, K.R. Kolesar, P.T., 2006, Devils Hole, Nevada, $\delta^{18} \mathrm{O}$ record extended to the mid-Holocene: Quaternary Research, 66, 202-212.

Yokoyama, Y., Esat, T.M., Lambeck, K., Fifield, L.K., 2000, Last ice age millennial scale climate changes recorded in Huon Peninsula corals: Radiocarbon, 42, 383-401.
Yokoyama, Y., Esat, T.M., Lambeck, K., 2001, Coupled climate and sea-level changes deduced from Huon Peninsula coral terraces of the last ice age: Earth and Planetary Science Letters, 193, 579-587

Manuscrito recibido: Agosto 24, 2009.

Manuscrito corregido recibido: Enero 11, 2010.

Manuscrito aceptado: Febrero 2, 2010. 